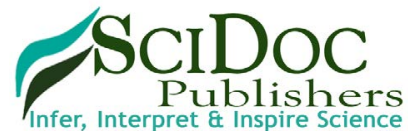

International Journal of Nano Studies \& Technology (IJNST) ISSN: $2167-8685$

\title{
Nanoparticle Based Combination Treatments for Targeting Multiple Hallmarks of Cancer
}

Review Article

VanDyke $\mathrm{D}^{1 \#}$, Kyriacopulos $\mathrm{P}^{2 \#}$, Yassini $\mathrm{B}^{1}$, Wright $\mathrm{A}^{1}$, Burkhart $\mathrm{E}^{1}$, Jacek $\mathrm{S}^{1}$, Pratt $\mathrm{M}^{1}$, Peterson $\mathrm{CR}^{1}$, Rai $\mathrm{P}^{1,2^{*}}$

${ }^{1}$ Department of Chemical Engineering, University of Massachusetts, Lowell, MA, USA.

${ }^{2}$ Biomedical Engineering and Biotechnology Program, University of Massachusetts, Lowell, MA, USA.

\#These authors (DV and PK) contributed equally to this work.

\section{Abstract}

Treatment of cancer remains one of the most challenging tasks facing the healthcare system. Cancer affects the lives of millions of people and is often fatal. Current treatment methods include surgery, chemotherapy, radiation therapies or some combinations of these. However, recurrence is a major problem. These treatments can be invasive with severe side effects. Inefficacies in treatments are a result of the complex and variable biology of cancerous cells. Malignant tumor cells and normal functioning cells share many of the same biological characteristics but the main difference is that in cancer cells there is in an overuse and over expression of these biological characteristics. These pertinent characteristics can be grouped into eight hallmarks, as illustrated by Hanahan and Weinberg. These characteristics include sustaining proliferative signaling, evading growth suppressors, resisting cell death, enabling replicative immortality, inducing angiogenesis, activating invasion and metastasis, reprogramming energy metabolism, and evading immune destruction. In order to provide a noninvasive, effective treatment, delivery methods must be explored in order to transport cytotoxic agents used for targeting the hallmarks of cancer in a safer and more effective fashion. The use of nanoparticles as drug delivery carriers provides an effective method in which multiple cytotoxic agents can be safely delivered to cancer tissue to simultaneously target multiple hallmarks. By targeting multiple hallmarks of cancer at once, the efficacy of cancer treatments could be improved drastically. This review explores the uses and efficacy of combination therapies using nanoparticles that can simultaneously target multiple hallmarks of cancer.

Keywords: Nanomedicine; Oncology; Combination Therapy; Drug Delivery; Hybrid Nanoparticles.

\section{Introduction}

The National Cancer Institute estimates that 1,685,210 new cases of cancer will be diagnosed in the United States in 2016, and of these projected cases, it is estimated that 595,690 will be fatal. It is also estimated that $39.6 \%$ of men and women will be diagnosed with cancer at some point during their lifetime [1]. Additionally, according to the American Cancer Society, based on data from 2005-2011, the five year survival rate for all cancers was $69 \%$. This is an increase from past years but is still a very low survival rate compared to other diseases, and some cancers still have survival rates of below 20\% [2]. These low figures illustrate a necessity for more efficient cancer treatment therapies.
Recently, observed survival rates have increased due to a better understanding of cancer biology, resulting in improved treatments and earlier diagnosis [3, 4]. Cancer death rates for men, women, and children under the age of 19 have decreased by 1.8 , 1.4, and 2.0 percent respectively from 2003-2012 [1]. Although there has been some success with traditional treatments such as surgery, chemotherapy and radiation therapy, these treatments can be cytotoxic and cause harm to normal tissue and the cancer mass, with some resulting in severe side effects. This cytotoxicity is one of the main reasons for the current inefficacies in treatment methods [3, 4].

Generally, tumor cells perform many of the same functions as normal cells. However, the difference between normal cells and

\footnotetext{
*Corresponding Author:

Prakash Rai,

Department of Chemical Engineering \& Biomedical Engineering and Biotechnology Program, University of Massachusetts, Lowell, MA, USA.

Tel: (978) 934-4971

Fax: (978) 934-3047

E-mail: prakash_rai@uml.edu

Received: June 30, 2016

Accepted: July 23, 2016

Published: July 27, 2016

Citation: Rai P et al., (2016) Nanoparticle Based Combination Treatments for Targeting Multiple Hallmarks of Cancer. Int J Nano Stud Technol, S4:001, 1-18.
} doi: http://dx.doi.org/10.19070/2167-8685-SI04001

Copyright: Rai $\mathbf{P}^{\circ}$ 2016. This is an open-access article distributed under the terms of the Creative Commons Attribution License, which permits unrestricted use, distribution and repro duction in any medium, provided the original author and source are credited. 
tumor cells is the lack of organization and the manipulation of regulatory processes in cancerous cells $[3,4]$. Because of these differences, there is a complexity to malignant tumors that can render normal treatment methods less effective, attributing to the low survival rates. These complexities were effectively addressed in Weinberg and Hanahan's groundbreaking cancer reviews in 2000 and 2011, which break down the characteristics of cancer into a set of eight hallmarks $[3,4]$.

These hallmarks of cancer include sustaining proliferative signaling, evading growth suppressors, resisting cell death, enabling replicative immortality, inducing angiogenesis, activating invasion and metastasis, reprogramming energy metabolism, and evading immune destruction $[3,4]$.

As a result of the publication of Weinberg and Hanahan's reviews, and the ineffectiveness of many traditional cancer treatments the focus of research has shifted towards understanding the tumor. By putting focus on the tumor, vital research has been conducted that can be used in creating more effective treatments of cancer. For example, it has been found that the tumor may be manipulated to either promote, or destroy itself [5]. In addition, it has been found that tumors are known to have hypoxic regions, which can contribute to chemoresistance [6]. This information is essential in both understanding the resistance of tumors to many chemo and radiotherapies and the development of new treatments.

As a result of the ineffectiveness and cytotoxicity of normal treatments in targeting the characteristics of the complex tumor microenvironment, new methods which can target those characteristics must be explored.

This review explores the hallmarks of cancerous tumors, the shortcomings of previous medication practices, the use of singletarget and multi-targeted nanoparticle approaches, and the apparent need for a multi-targeted approach that targets multiple hallmarks of cancer.

\section{Hallmarks of Cancer}

In Hanahan and Weinberg's groundbreaking reviews, eight core hallmarks of cancer were illustrated. These hallmarks include:

1. Sustaining Proliferative Signaling- In normal cells, proliferation signals will be activated only in the event of developmental growth or injury. However, in cancer cells, these proliferative signals are constantly being used to form rapidly growing tumor structures $[3,4]$.

2. Evading Growth Suppressors- Healthy cells inhibit unwanted proliferation by sending growth suppressors to cease cell growth. In cancer cells, these growth suppressors are resisted, and thus continuing rapid and proliferative cell growth continues [3, 4].

3. Resisting Cell Death- As time passes, cell functions become corrupted. When this happens, cells should perform a form of cell suicide, with apoptosis being the most desirable form. However, cancer cells resist and inhibit cell suicide. This inhibition leads to the protection of improperly functioning cells $[3,4]$.

4. Enabling Replicative Potential- Cancer cells have the ability to replicate infinitely by maintaining the length of their genetic sequences. Usually, as a cell divides, the genetic sequence will shorten. This occurs because telomerase, an enzyme present in the cell's nucleus, protects the ends of the nucleotide sequences during replication $[3,4]$.

5. Inducing Angiogenesis- New cells need blood vessels to transport nutrients and drain waste material. This occurs through the use of Vascular Endothelial Growth Factor, (VEGF). In cancer cells, VEGF is over-abundant, thus promoting the formation of improper blood vessels, which are characteristic of cancer cells [3, 4].

6. Activating Invasion and Metastasis- Malignant tumor cells have the ability to separate themselves from the original tumor site and search for new areas to invade [3, 4].

7. Reprogramming Energy Metabolism- Every cell's energy is obtained through anaerobic or aerobic energy production by converting glucose to ATP. However, in cancer cells, glucose transporters are upregulated and aerobic glycolysis is preferred. These transporters can be used to produce new nucleosides or amino acids, which are used in cell proliferation [4].

8. Evading Immune Destruction-In normal cells, T-lymphocytes monitor cells by observing cell markers. If these markers indicate that a cell is not behaving properly, the T-lymphocytes can puncture these misbehaving cells. However, these markers are poorly expressed in cancer cells, and thus they avoiding immune destruction [4].

\section{Use of Nanoparticles}

One of the main challenges facing cancer treatment is reducing the collateral damage to normal tissues from cytotoxic therapies. The use of nanoparticles as drug carriers is becoming increasingly common in the development of a safe and effective treatment method. Nanoparticles have the ability to use a single structure to transport hydrophobic and hydrophilic molecules to specific target areas. Also, nanoparticles are attractive for cancer treatment due to their size, thus giving them the ability to travel through the poorly made, leaky vasculature of a malignant tumor, yet large enough to escape immediate clearance [7]. Additionally, nanoparticles have the ability to be loaded with multiple cancer medications and conjugated with targeting moieties that allow for the targeting of various hallmarks of cancer $[3-5,8,9]$. Nanoparticles provide a targeted combination treatment method that allows for the safe transport and release of cytotoxic agents to the core of the tumor microenvironment.

Nanoparticles allow for the safe transport and release of cytotoxic agents, increasing the therapeutic index, the ratio of the amount of a therapeutic agent that will give a positive effect versus the amount of a therapeutic agent that may cause a toxic or deadly effect [10]. For example, the drug paclitaxel relies on the use of Kolliphor EL to increase drug solubility during intravenous infusion in patients. However, Kolliphor EL is a highly toxic substance that leaches plasticizers and can lead to severe anaphylaxis, abnormal lipoprotein patterns, irreversible sensory neuropathy, histamine disease, hyperlipidemia, and the aggregation of erythrocytes. To decrease cytotoxicity, paclitaxel was prepared in an albumin-bound suspension, which serves as a nanoparticle drug delivery carrier. This new formulation, known as Abraxane, delivers the same amount of cancer treatment safely without many of the harmful side effects of normal paclitaxel treatment [11]. 
In addition, nanoparticles offer the ability to transport multiple therapeutic agents simultaneously [10]. This allows for multiple hallmarks to be targeted simultaneously, resulting in faster, safer and more targeted tumor destruction [10]. Also, by using multiloading methods, the tumor core can be diminished at a higher rate. By using simultaneous drug delivery, tumors can be destroyed in a safer and more targeted fashion, and multiple hallmarks can be targeted at once [12].

Because of nanoparticles ability to transport multiple drugs simultaneously, traditional therapies can be combined with nanomedicines to increase drug response and reduce toxicity. In a 2008 study, Abraxane was administered with Gemcitabine. Both are apoptosis inducers, but Abraxane affects cell division while Gemcitabine blocks DNA replication. It was observed that $50 \%$ of all patients in this Phase II study had a confirmed response. Of the total number of patients, $8 \%$ observed a complete response and $42 \%$ observed a partial response. Also, it was observed that the therapy's toxicity was well tolerated [13].

Therapeutic drugs in nanoparticle constructs can also be combined with photosensitizers for drug delivery and treatment by photodynamic therapy (PDT). PDT uses photosensitizing agents, that when exposed to light of a certain wavelength produce a radical oxygen species that kills neighboring cancer cells [14]. This combination of chemotherapeutics and PDT provides an effective treatment method that can be used to target multiple hallmarks of cancer.

\section{Passive versus Active Targeting of Nanoparticles}

In addition to being able to transport multiple drugs simultaneously, nanoparticles either have the ability to actively or passively target tumor sites. Passive targeting involves taking advantage of the poorly formed vasculature, and the Enhanced Permeability and Retention (EPR) effect to transport nanoparticles and treatments more effectively to the tumor core, tumor vasculature, and tumor microenvironment [7-9]. Tumor vasculature is often synonymous with leaky blood vessels and poor lymphatic drainage of the vasculature. Because of this poorly made vasculature, it is difficult for preexisting treatment methods to effectively penetrate the tumor core [7]. These imperfections in the tumor vasculature may limit normal treatments but can assist in the transportation of nanoparticles to the tumor core through the use of the EPR. In a 2013 study, photo-immunotherapies were used to increase the EPR effect in the tumor vasculature. By using a photosensitizer conjugate, larger amounts of nano-sized agents $(10-200 \mathrm{~nm})$ were absorbed into the tumor bed. Therefore, the EPR effect can be used to increase the effectiveness of a nanoparticle in targeting the tumor core [15].

The use of the EPR effect to transport nanoparticles to the tumor core is one common example of passive targeting of nanoparticles. As mentioned above, nanoparticle treatment methods can either passively or actively target cancerous tumors. This targeting of the tumor site, either actively or passively, is one of the key benefits of using nanostructures. As described above, the EPR effect exploits the imperfect tumor blood vessels and poor lymphatic drainage which allows for the natural accumulation of nanostructures and other molecules towards the tumor. This natural accumulation of macromolecules is referred to as passive targeting, as there are no active targeting agents involved for molecule accumulation at the tumor site. One of the biggest issues of passive targeting comes from the complexity of the tumor environment. Every tumor is going to be unique in its own way and therefore the nanostructure and use of the EPR must be altered. In future clinical trials this would involve fully assessing the tumor of a patient before using means of passive transport to target the cancerous tumor. This adds additional time and costs that must be taken into consideration when considering using nanoparticles for passive targeting. The positive side of passive targeting is that no surface modifications need to be made, like in the case of active targeting [16].

Active targeting, involves the use of targeting moieties such as monoclonal antibodies (mAb), proteins, polymers, or aptamers to target specific types of cancerous cells. Using targeted nanoparticles for active targeting has become one of the many uses of nanostructures for drug delivery, but there is still debate on the efficacy of active treatments over passive treatments in vivo. Although active targeting allows for specific targeting of receptors on the tumor cells there has been evidence that in vivo active targeting may not be as successful as in vitro active targeting [16]. One study found that active targeting did in fact promote cellular uptake, but it also affected the biodistribution of the drug carrier and showed unfavorable tumor partitioning. The study concluded that targeting moieties were only successful in vitro but was not conclusive in vivo. This is something that may vary depending on the nanostructure and targeting moiety being used but is something that should be considered when deciding between the active or passive targeting of tumor sites [17]. The effects of active transport through the use of targeted nanoconstructs is discussed in further sections involving drug loaded nanoparticles. These nanoconstructs used for active targeting will often be referred to as targeted drug delivery methods. Overall, the targeting of tumor sites by either active or passive targeting is one of the main reasons nanoconstructs have been viewed as popular drug delivery carriers.

Nanoparticles provide a biocompatible, targeted treatment method that allow for high encapsulation of drug molecules, small concentrations of premature drug release, and have the ability of controlled drug release making nanoparticles a strong platform for the targeting of multiple cancer hallmarks. A depiction of a general, targeted (active targeted), and non-targeted (passive targeted), drug loaded nanostructures can be seen in Figure 1.

\section{Goals of Nanoparticle Design}

With new types of nanoparticles being produced every day, there are some immutable concepts that must be recognized when synthesizing new nanoparticles.

As stated in a 2013 perspective paper by Grodzinski and coworkers, six "tenets" must be followed when synthesizing nanoparticles. These tenets include understanding the nanoparticle's biological target, and avoiding biological hurdles that may impair the transport of nanoparticles. Also, nanoparticles are not tablets; they are complex chemicals that can be conjugated with ligands for better targeting. In other words, the chemical mechanism of each nanoparticle must be understood. The fifth tenet illustrates that technological advances must be used in a practical sense. Of- 
Figure 1. Drug loading strategies in a targeted nanostructure. (A) General, unloaded, nanoparticle. (B) Single-drug loaded nanoparticle. (C) Multi-loaded nanoparticle. (D) Multi-loaded nanoparticle with targeting ligand on the surface.

A.

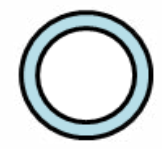

B.

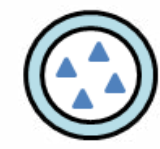

C.

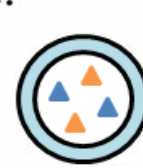

D.

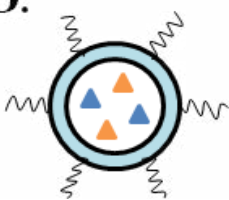

Key

$\Delta$ Drug A

Drug B

乡 Targeting Ligand ten, nanoparticles are made on a lab scale, so if these nanoparticles are to be made commercially, the process of developing them must be scaled up to accommodate mass production. The final tenet is related to the previous tenet, but states that an effective nanotherapy must be economically viable. If the production of these nanoparticles is not economically viable, then the business will ultimately fail and is not worth pursuing $[18,19]$.

In addition, in order for nanoparticles to be a successful drug delivery system, according to Slowing, et al., there are five prerequisites that must be met. These prerequisites include biocompatibility, the ability for high encapsulation of drug molecules, limited premature release of drug molecules, site directing ability, and the controlled release of drug molecules [20]. These five factors must be taken into consideration during nanoparticle design so that the nanoparticle serves as an efficient drug delivery system.

Also, as stated in a 2012 article written by Hall, et al., although nanoparticles are not smaller than the drugs they encapsulate, they are more monodisperse and have the ability to avoid agglomeration. It should be noted that this change in size may lead to a different physiological response, depending on the drug [21]. These factors must be understood and referenced while synthesizing new and more intricate nanomedicines.

It is also important when targeting multiple hallmarks using nanoparticles that the correct combinations are selected and scheduling of the individual therapies is understood and evaluated. For example, it might not be very effective to target the evasion of antiproliferative signals with proliferative signals. A more effective dosing method may include targeting proliferative cells/signaling with an anti-vasculature medication. This is another concept to keep in mind during the process of designing a nanoparticle combination treatment method.

The following sections of this review will focus on the various types of nanoparticles, including liposomes, polymeric nanoparticles, dendrimers, mesoporous silica nanoparticles, metallic nanoparticles, and nanohybrids and their abilities for drug loading and combination treatments to target multiple hallmarks of cancer. Depictions of some of these nanoconstructs can be seen in Figure 2. Additionally, many of the drug molecules mentioned in the following sections, and their targeted hallmarks, are summarized in Table 1.

\section{Types of Nanoparticles and Multi-Loading Tech- niques}

\section{Liposomes}

Liposomes are cell-like structures composed of a lipid bilayer. Liposomes are advantageous because they have both hydrophilic and hydrophobic regions. The base component of a liposome is a single lipid that has a hydrophilic head, and one or more hydrophobic tails. The hydrophilic outer layer allows liposomes to be transported throughout the body, while hydrophobic drugs or markers can be loaded in the hydrophobic transmembrane section. By using this construct, drugs can be precisely and effectively transported to a specific target site $[22,23]$. In addition, liposomes have been seen to bypass the issue of multidrug resistance seen in some cancerous cells [24]. By using liposomes to transport cytotoxic drugs, as it will be described below, safer and

Figure 2. Various nanoconstructs used for combination therapy of cancer. (A) Liposome, with its characteristic lipid bilayer. Niosomes exhibit a similar structure except are composed of surfactants rather than phospholipids. (B) Polymeric nanoparticle, composed of multiple monomers. (C) Dendrimer, with its branched structure of monomers. (D) Mesoporous silica nanoparticle, with porous structure used for drug loading. (E) Quantum dots. (F) Single-wall carbon nanotube. (G) Metallic nanoparticles, such as gold nanoparticles. $(\mathrm{H})$ Nanohybrid construct, composed of a silica core and a thin gold shell.

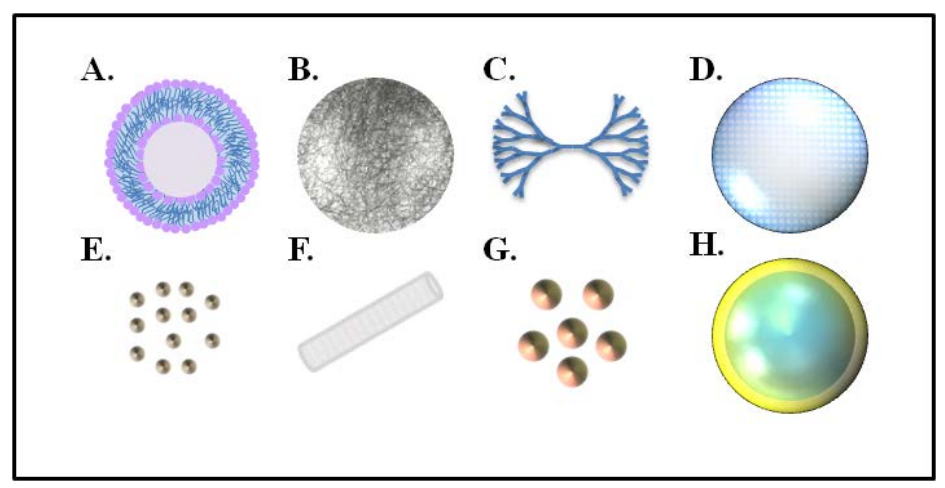


Table 1. Drug Information for common chemotherapy drugs or biologics and the hallmarks of cancer they target. All drug information obtained from National Cancer Institute Drug Dictionary [90].

\begin{tabular}{|c|c|c|c|c|}
\hline Drug & Drug Class & Uses & Treatment Mechanism & Targeted Hallmark(s) \\
\hline Paclitaxel & Plant alkaloid & $\begin{array}{l}\text { Breast, ovarian, lung, } \\
\text { bladder, prostate, } \\
\text { melanoma, esophageal } \\
\text { cancers }\end{array}$ & $\begin{array}{l}\text { Mitotic inhibitor and } \\
\text { microtubule inhibitor }\end{array}$ & $\begin{array}{l}\text { Resisting cell death, and } \\
\text { infinite replicative potential }\end{array}$ \\
\hline Gemcitabine & Antimetabolite & $\begin{array}{l}\text { Pancreatic, lung, blad- } \\
\text { der, breast, and ovarian } \\
\text { cancers }\end{array}$ & $\begin{array}{l}\text { Pyrimidine antagonist, } \\
\text { inhibits cell division }\end{array}$ & $\begin{array}{l}\text { Resisting cell death, and } \\
\text { infinite replicative potential }\end{array}$ \\
\hline $\begin{array}{l}\text { Doxorubicin } \\
\text { (DOX) }\end{array}$ & $\begin{array}{l}\text { Anthracycline } \\
\text { antitumor } \\
\text { antibiotic }\end{array}$ & $\begin{array}{l}\text { Leukima, breast, endo- } \\
\text { metrial, gastric, head/ } \\
\text { neck, liver, kidney, } \\
\text { ovarian, lung, and thyroid } \\
\text { cancers }\end{array}$ & $\begin{array}{c}\text { Acts during multiple } \\
\text { phases of cell cycle to } \\
\text { interact with DNA, induc- } \\
\text { ing apoptosis }\end{array}$ & $\begin{array}{l}\text { Resisting cell death, and } \\
\text { infinite replicative potential }\end{array}$ \\
\hline Daunorubicin & $\begin{array}{l}\text { Anthracycline } \\
\text { antitumor } \\
\text { antibiotic }\end{array}$ & $\begin{array}{l}\text { Leukemia, and lung } \\
\text { cancer }\end{array}$ & $\begin{array}{c}\text { Interacts with DNA in } \\
\text { order to induce apoptosis } \\
\text { and inhibits cell replica- } \\
\text { tion }\end{array}$ & $\begin{array}{l}\text { Resisting cell death, and } \\
\text { infinite replicative potential }\end{array}$ \\
\hline 6-mercatopurine & Antimetabolite & $\begin{array}{l}\text { Lymphoma, and leuke- } \\
\text { mia }\end{array}$ & $\begin{array}{l}\text { Purine antagonist, inter- } \\
\text { feres with the cell cycle }\end{array}$ & $\begin{array}{l}\text { Resisting cell death, and } \\
\text { infinite replicative potential }\end{array}$ \\
\hline Irinotecan & Plant alkaloid & Colon, and rectal cancer & $\begin{array}{l}\text { Topoisomerase inhibitor, } \\
\text { effecting DNA and cell } \\
\text { replication }\end{array}$ & $\begin{array}{l}\text { Resisting cell death, and } \\
\text { infinite replicative potential }\end{array}$ \\
\hline Floxuridine & Antimetabolite & $\begin{array}{c}\text { Colon, kidney, and stom- } \\
\text { ach cancers }\end{array}$ & $\begin{array}{l}\text { Pyrimidine antagonist, } \\
\text { interferes with cell cycle }\end{array}$ & $\begin{array}{l}\text { Resisting cell death, and } \\
\text { infinite replicative potential }\end{array}$ \\
\hline Bevacizumab & $\begin{array}{l}\text { Monoclonal } \\
\text { antibody }\end{array}$ & $\begin{array}{l}\text { Colon, rectal, lung, and } \\
\text { breast cancers }\end{array}$ & $\begin{array}{l}\text { VEGF inhibitor, inhibits } \\
\text { angiogenesis }\end{array}$ & Angiogenesis \\
\hline Cabozantinib & Targeted therapy & Thyroid cancer & $\begin{array}{l}\text { Inhibits tyrosine kinas- } \\
\text { es, blocks pathways that } \\
\text { promote cell division }\end{array}$ & $\begin{array}{c}\text { Proliferative signaling, } \\
\text { evading growth suppres- } \\
\text { sors, and infinite replicative } \\
\text { potential }\end{array}$ \\
\hline Methotrexate & Antimetabolite & $\begin{array}{l}\text { Leukemia, osteosarcoma, } \\
\text { head/neck, lung, and } \\
\text { stomach cancers }\end{array}$ & $\begin{array}{l}\text { Causes folic acid defi- } \\
\text { ciency which leads to cell } \\
\text { death }\end{array}$ & Resisting cell death \\
\hline $\begin{array}{c}\text { Combretastatin } \\
\text { A4 }\end{array}$ & $\begin{array}{c}\text { Vascular } \\
\text { disrupting agent }\end{array}$ & $\begin{array}{l}\text { Thyroid, ovarian, and } \\
\text { lung cancers }\end{array}$ & $\begin{array}{c}\text { Disrupts tumor vascu- } \\
\text { lature, induces tumor } \\
\text { necrosis }\end{array}$ & $\begin{array}{c}\text { Angiogenesis, and resisting } \\
\text { cell death }\end{array}$ \\
\hline Verapamil & $\begin{array}{l}\text { Calcium channel } \\
\text { blocker }\end{array}$ & $\begin{array}{l}\text { Hypertension, angina } \\
\text { pectoris, and cardiac } \\
\text { arrhthymia }\end{array}$ & $\begin{array}{l}\text { Aids in silencing multi- } \\
\text { drug resistance }\end{array}$ & Resisting cell death \\
\hline $\begin{array}{c}\text { 17-AAG } \\
\text { (tanespimycin) }\end{array}$ & $\begin{array}{l}\text { Antitumor } \\
\text { antibiotic }\end{array}$ & $\begin{array}{l}\text { Leukemia, and kidney } \\
\text { cancer }\end{array}$ & $\begin{array}{l}\text { Inhibits the tumor } \\
\text { promoting protein Hsp90 }\end{array}$ & $\begin{array}{c}\text { Evading growth } \\
\text { suppressors, and } \\
\text { proliferative signaling }\end{array}$ \\
\hline Rapamycin & Macrolide & $\begin{array}{c}\text { Lymphoma, Kaposi's } \\
\text { sarcoma }\end{array}$ & $\begin{array}{c}\text { Enhances immune re- } \\
\text { sponse to tumor targeting }\end{array}$ & $\begin{array}{l}\text { Evading growth suppres- } \\
\text { sors, and evading immune } \\
\text { destruction }\end{array}$ \\
\hline Wortmannin & $\begin{array}{c}\text { Steroid } \\
\text { metabolite }\end{array}$ & $\begin{array}{c}\text { Ovarian, head/neck, } \\
\text { cervical, and lung cancers }\end{array}$ & $\begin{array}{l}\text { Inhibits cell survival sig- } \\
\text { naling pathways }\end{array}$ & $\begin{array}{l}\text { Proliferative signaling, and } \\
\text { invasion and metastasis }\end{array}$ \\
\hline $\begin{array}{c}\text { Camptothecin } \\
\text { (CPT) }\end{array}$ & Plant alkaloid & Colon, rectal cancers & $\begin{array}{l}\text { Topoisomerase inhibitor, } \\
\text { inhibits cell division }\end{array}$ & $\begin{array}{l}\text { Resisting cell death, and } \\
\text { infinite replicative potential }\end{array}$ \\
\hline $\begin{array}{l}\text { All-trans retinoic } \\
\text { acid }\end{array}$ & Retinoid & Leukemia & $\begin{array}{l}\text { Controls cell growth and } \\
\text { differentiation }\end{array}$ & $\begin{array}{l}\text { Evading growth } \\
\text { suppressors, and infinite } \\
\text { replicative potential }\end{array}$ \\
\hline
\end{tabular}


more targeted delivery can be achieved.

Liposome Production and Loading: Various processes can be used to create liposomes. Liposome synthesis commonly uses a combination of lipids that are mixed together resulting in polydisperse, multilamellar liposomal constructs. In order for liposomes to be effective they must be monodisperse and unilamellar, which is achieved through either sonication, or more commonly, extrusion. Sonication involves the use of sound waves to evenly disperse particles, while extrusion involves the use of mechanical energy to push fluid through a membrane of a specific pore size resulting in unilamellar, monodisperse liposomes.

One common synthesis method involves the heating of organic material, as explained by Colas, et al., In this study, cholesterol, dicetyl phosphate, dipalmitoyl phosphatidylcholine, Phospholipon $90 \mathrm{H}$, Phospholipon $100 \mathrm{H}$, and stearylamine are mixed at varying concentrations and added to a preheated mixture of nisin and glycerol (heated for $5 \mathrm{~min}$ at $60^{\circ} \mathrm{C}$ ). The purpose of heating the organic material in the presence of nisin and glycerol was to effectively load nisin (a protein used to inhibit gram-positive bacteria) into the liposomal construct. This mixture was stirred for an additional $45-60$ minutes at $60^{\circ} \mathrm{C}$ under a nitrogen atmosphere. After stirring, the aqueous layer is removed and then the liposomes areextruded to produce unilamellar, monodisperse liposomes.This study illustrated an efficient synthesis method and confirmed that large molecules, such as proteins can be loaded into a liposome and released in a controlled fashion $[22,23]$.

Once liposomes are produced, they can be coated with polymeric compounds for a variety of reasons. Most importantly, these surface polymers can increase the liposomes' biocompatibility and biodegradability. For example, it has been seen that by coating a liposome with polyethylene glycol (PEG), the liposomal nanostructure can circulate throughout the body without any immune response. This type of liposome is often referred to as a Stealth Liposome [25]. In addition, polymers on the surface, such as PEG, can also be used for creating a targeted delivery method by using a biconjugated polymer. One end of the polymer will be used to bind to the surface of the liposome and the other end used to target a cancerous tumor.

In addition to polymers, targeting molecules such as monoclonal antibodies ( $\mathrm{mAb}$ ), proteins, aptamers, etc. can be used as successful targeting agents. As well as being able to attach targeting agents on the outer membrane, liposomes possess the ability for targeting molecules to be conjugated either inside the liposome, or in the transmembrane section of the liposome. Therefore, liposomes offer the option for a variety of targeted treatments that can be used to target a ligand, receptor, protein, etc. [22, 23].

One example of a targeted liposomal treatment method was seen in a recent study in which gemcitabine encapsulated liposomes were conjugated with hyaluronan for targeting breast cancer stem cells. Hyaluronan serves as an appropriate targeting method for breast cancer stem cells because it is a primary ligand for CD44 surface markers, and gemcitabine is administered as a chemotherapeutic, that serves as an antimetabolite used to reduce cell division of cancerous cells. In addition, hyaluronan is naturally occurring in the body, increasing the biocompatibility of the nanostructure. This study showed promise for hyaluronan conju- gated liposomes in targeting breast cancer stem cells both in vitro and in a xenograft mouse model [26].

In addition to targeting specific ligands or receptors, liposomes can also be used to target specific cells. Liposomes are freely absorbed into a cell through the act of phagocytosis. Once inside the cell, the liposomal construct breaks down releasing its inner contents to be freely distributed within the cell. These materials could then either target certain organelles or be activated within the cell. This method of liposomal drug delivery can administer potentially cytotoxic agents in a safe and controlled manner [23, 24].

Drug loading of liposomes has been very successful and some liposome based nanomedicines have even been successful clinically. One example is Doxil, which consists of the encapsulation of doxorubicin (DOX) inside a liposome construct [27]. The use of a liposomal construct allows for the safe transport of DOX, normally a cytotoxic chemotherapy medication. Another approved nanomedicine is DaunoXome, which much like Doxil involves a chemotherapy drug (daunorubicin) encapsulated inside of a liposome [28]. Both DOX and daunorubicin interact with the DNA of a cell, inducing apoptosis. These are just some of the many examples of studies that have successfully drug loaded liposomal nanoparticles.

Liposomes have also been widely used for PDT, one study showed that liposomes are efficient carriers of photosensitizers to angiogenic endothelial cells. From there PDT can be used to target angiogenesis of cancerous tumors [29].

The above treatment methods involve single treatment methods using liposomal nanoconstruct, the next section will focus on combination treatments using liposomes and various therapeutic agents, from chemotherapy drugs to photosensitizers used for PDT.

\section{Multiple Drug Loading of Liposomes for Treating Cancer:} While using one loading method to target cancer may be effective, the efficacy of nanoparticles increases when structures are loaded with multiple targeting agents or medicines. Liposomes loaded with multiple therapeutic agents prove to be more effective than liposomes loaded with a single drug. By having multiple therapeutic agents loaded in one nanoparticle, multiple hallmarks of cancer can be targeted at once.

Multiple studies have focused on loading liposomes with multiple chemotherapeutic agents in order to improve efficacy of treatments $[30,31]$. One study found that by loading a liposome with multiple drugs, instead of one, Jurkat and Hut 76 T-Cell lymphoma can be treated more effectively due to a heightened cytotoxicity of the liposomes. Additionally, it was determined that higher cytotoxicity against Jurkat and Hut 76 T-cell lymphoma was observed when liposomes were dual loaded, as compared to mono-loading of the same drugs. This study focused on the use of daunorubicin and 6-mercaptopurine, two chemotherapeutic agents that initiate apoptosis by targeting specific steps of the cell cycle [12].

Another study reviewed in the same paper, used a liposomal construct to deliver siRNA and chemotherapeutics in a combined 
treatment method. This method illustrated that the siRNA targeted specific mRNA sequences, thus disrupting the unlimited replicative potential of the cancerous cells, one of the hallmarks of cancer. Additionally, like most chemotherapeutic methods, the chemotherapeutics used would target actively proliferating cells to limit the replicative potential of cancerous cells. Therefore, this study exhibits just one example of how liposome nanostructures can be used not only to carry chemotherapeutic drugs, but also other molecules that can be used for cancer treatment, such as siRNA [12].

Furthermore, a Phase I study was described by Batist, et al., showed the effects of multi-loading of chemotherapeutic agents. This Phase I study involved the encapsulation of two different drugs, irinotecan and floxuridine, in a single liposomal construct. This method of combination chemotherapy proved more effective than traditional treatments by safely delivering these cytotoxic agents to the tumor, where they acted as effective, anti-proliferative and anti-metastatic agents to the cancerous cells. The purpose of the study was to determine the optimum-loading ratio for each chemotherapy medication and once administered to patients, it was found that the correct dosage to initiate an anticancer response was 210 units $/ \mathrm{m}^{2}$ of a 1:1 ratio of irinotecan and floxuridine.After treatment, it was determined that three patients received a partial anticancer response, 21 patients achieved a stable disease, and six patients had a progressive disease response. Although the response was not completely effective, it shows promise for the use of the combination treatment involving multiple chemotherapeutic agents in a liposomal construct [32].

In addition to the loading of multiple drugs in a liposomal construct, chemotherapy drugs and photosensitizing agents can be combined in order for the delivery of chemotherapeutic agents and treatment by PDT. A 2015 study used the encapsulation of the photosensitizer benzoporphyrin derivative monoacid A (BPD) and bevacizumab, the anti-VEGF monoclonal antibody that helps to inhibit angiogenesis, inside a single liposomal construct for treatment of pancreatic cancer. It was found that the combination treatment method enhanced drug delivery extra- and intracellularly and decreased cytotoxicity in vitro. Additionally, in an in vivo subcutaneous mouse model of pancreatic ductal adenocarcinoma the combination treatment enhanced overall tumor reduction [33]. It is seen that through the use of liposomal constructs chemotherapeutic drugs can be delivered more efficiently and the efficacy of photodynamic therapy can be increased, allowing for more effective combination treatments.

Another recent study described by Spring et al., focused on a photoactivable liposomal nanostructure that could be used for light-induced cytotoxicity as well as sustained, photoinitiated release of inhibitors used to target tumor regrowth and treatment escape pathways. The nanoliposomes were used to encapsulate cabozantinib, a multikinase inhibitor, and were synthesized with the photosensitizer BPD in the lipid bilayer. This provided for a combination treatment that could be used for simultaneously targeting multiple hallmarks of cancer, including tumor repression, and inhibition of treatment escape signaling pathways. This liposomal nanoparticle not only allowed for near-infrared tumor suppression through the use of BPD, but also allowed for the photoinitiated drug release of cabozantinib, limiting the cytotoxicity of systemic drug exposure. This combination treatment was seen to prolong tumor growth in two mouse models, and was also seen to inhibit metastatic escape. The basics of this study and its findings are illustrated in Figure 3 [34].

Above are only a select few of the multitude of current combination treatment studies involving liposomal nanostructures. Liposomal nanostructures allow for the combination of multiple chemotherapeutic drugs, as well as the combination of chemotherapeutic drugs with photosensitizing agents. As seen, combination treatments can increase the efficacy of normal treatment methods by the simultaneous targeting of multiple hallmarks of cancer.

\section{Polymeric Nanoparticles}

Polymers are macromolecules composed of multiple smaller molecules, referred to as monomers that are chemically linked together in repeating subunits. There are both naturally occurring and artificially produced polymers, and polymers are extremely versatile materials used for a variety of applications. Polymers are especially useful in nanomedicine, where polymers can be used as a drug delivery vehicle that allow for the gradual, sustained release of therapeutic agents.

Additionally, polymers are attractive drug carriers because of their biocompatibility and biodegradability. One common polymer used in nanoparticle design is PEG due to its biocompatibility. One example of PEG's biocompatibility was seen when conjugated with liposomes. Due to the conjugation of liposomes with PEG, as discussed earlier, there was no immune response, allowing liposomes to circulate through the body. It has also been described that by using PEG, either as a surface agent on a nanostructure, or as the base component of a polymeric nanostructure, there is an extended blood-circulation time [25]. These are just a few of the key characteristics of PEG in nanoparticle design.

Another polymer common in nanoparticle design is hyaluronic acid (HA) which was discussed in an earlier section where it was used as a targeting agent on the surface of a liposome. HA is a naturally occurring chemical in the human body, located mainly in joints, used as a natural lubricant. This exemplifies both the biocompatibility and biodegradability properties of HA. In addition HA has self-assembling properties, and the ability to deliver hydrophobic cancer medications. By using the self-assembling properties of hyaluronic acid, therapeutic drugs can be carried by this soluble polymer making it a viable drug carrier $[35,36]$.

It is important to understand that PEG and hyaluronic acid are only a few examples of polymers that can be used to deliver therapeutic medications to a target site within the human body. The following sections will discuss other types of polymers and how they can be used not only to target specific areas of the body, and treat different ailments, but also their ability be loaded with multiple medications to treat cancerous tumors [37].

Polymeric Nanoparticle Production and Loading: As stated previously, there are a variety of polymers that currently exist, meaning that there are a variety of polymeric nanostructures that can be synthesized. One common synthesis method is through the use of free-radical polymerization to produce a copolymer of $\mathrm{N}$-isopropylacrylamide with $\mathrm{N}$-vinyl-2-pyrrolidone [38]. Free 
Figure 3. A photoactivable multi-inhibitor liposome (PMIL) for targeting multiple hallmarks of cancer. (A) Synthesis of a PMIL loaded with a benzoporphyrin derivative (BPD) in the lipid bilayer and cabozantinib (XL184) loaded in the liposomal core. (B) Treatment mechanism of PMILs, illustrating the multiple targeted hallmarks. (C) Fraction of residual tumor in a mice model 19 days after treatment. PMILs had greater tumor reduction compared to free XL184, XL184 loaded in a liposomal construct, BPD loaded in a liposomal construct, and a combination of liposomes with BPD and liposomes with XL184. (D) Comparison of the different methods mentioned in (C) in the number of cancer cells that exhibited liver and lymph node metastasis after treatment. PMILs had the least number of cancer cells exhibiting metastasis after treatment

[32]. Reproduced from [32] by permission of Nature Publishing Group (NPG).

A. $a$

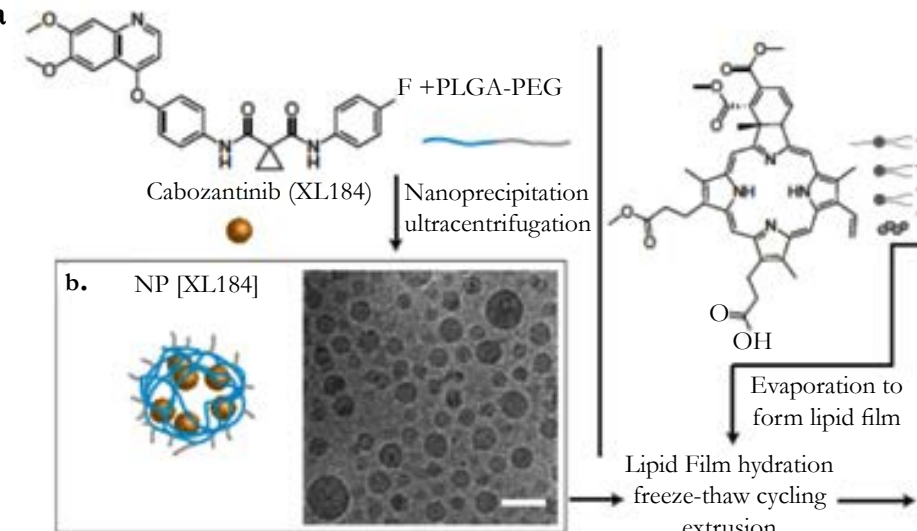
extrusion
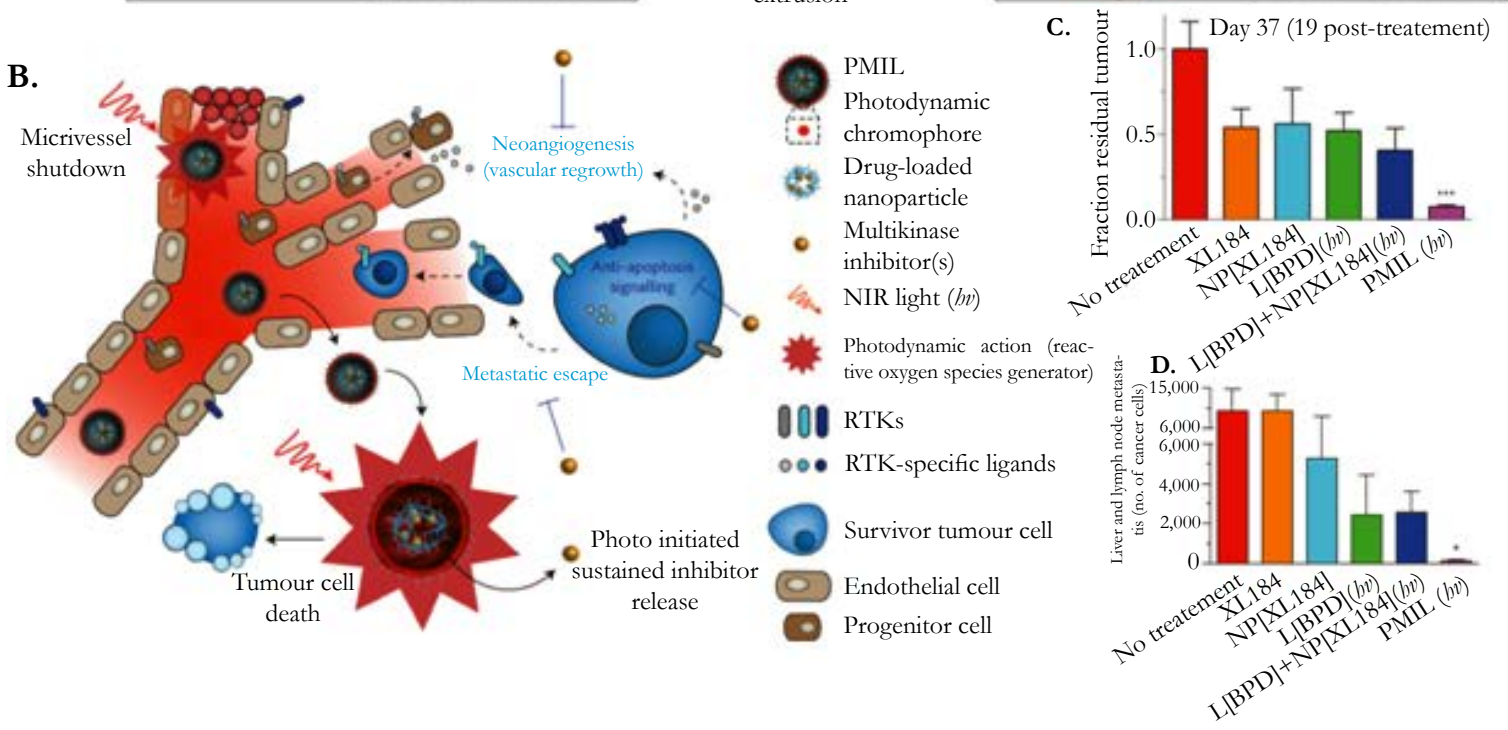

radical polymerization requires the use of monomers for the synthesis of polymers. Through free radical polymerization monomers are grouped together into a polymer by the successive addition of monomers through the use of initiator molecules. Once monomers are grouped, they can be emulsified, suspended, or placed in bulk solution.

Polymers such as PLA and PLGA have also been used in nanomedicine developments. PLA and PLGA are desirable because of their high degree of biodegradability. In an aqueous environment, PLA hydrolyzes into lactic acid while PLGA hydrolyzes into lactic acid and glycolic acid, which are both naturally occurring substances within the human body. To create PLA/PLGA polymeric nanoparticles, solvent displacement methods, emulsification, solvent evaporation, or salting out can be employed. If drug loaded, PLGA and PLA also offer a steady, controlled release of the drug. Additionally, much like PEG, PLA and PLGA can mask the hydrophobicity of many therapeutic agents. By encapsulating cytotoxic and hydrophobic cancer medications in PLA and PLGA polymeric nanoparticles there can be a controlled, safe approach of drug delivery $[31,35]$.

Once synthesized, polymers are then conjugated with targeting molecules or loaded with therapeutic agents for treatment applications $[39,40]$. Conjugating polymeric nanoparticles with markers lends to a more precise therapy method. Biomarkers are commonly used as targeting moieties including the EGFR-2 (erbB2/ HER2) and folic acid receptors. These two markers are often sought out due to their over expression seen in cancerous tumors. [41]. By targeting specific receptors there is a reduced chance of cytotoxic agents harming surrounding tissue. One study found that by targeting the over expression of folic acid in $\mathrm{KB}$ tumors that only cancerous cells were targeted. When these targeted polymeric nanostructures were encapsulated with the chemotherapeutic methotrexate, the dose near the tumor site increased after four days, while it usually decreases after two hours of treatment. This study illustrated that harmful, cytotoxic agents can be transported safely and effectively to a tumor without harming surrounding tissue [42].

A recent study used the encapsulation of siRNA into a polymeric nanoparticle structure in order to silence multiple endothelial 
genes. It has been found that dysfunctional endothelium contributes to the most amount of diseases in the body and that siRNA can be used to treat endothelial cells by the simultaneous silencing of multiple genes. This study found that polymeric nanoparticles efficiently transported siRNA to endothelial cells. In mouse models it was found that siRNA loaded polymeric nanoparticles modified endothelial function with respect to vascular permeability, emphysema, primary tumor growth and metastasis. This study illustrates that polymeric nanoparticles can be used in the loading of molecules other than chemotherapeutic agents including siRNA which can be used for treatment of cancer and a variety of other diseases [43].

By encapsulating a drug in a polymer construct, safer and more controlled release methods can be explored. In addition, targeting moieties can be conjugated on the surface of these polymer constructs for precise cell targeting. If multiple medications and targeting molecules are coupled with polymeric nanoparticles, then a more effective cancer treatment can be achieved that can be used to simultaneously target multiple cancer hallmarks.

\section{Multiple Drug Loading of Polymeric Nanoparticles for} Treating Cancer: Loading polymeric constructs with multiple cancer medications can increase the efficacy of treatment methods for malignant tumors. By targeting multiple hallmarks simultaneously, cancerous cells, which can become resistant to singledrug loaded methods, may not be able to adapt to a variety of attacks at once. Polymeric nanoparticles can transport a variety of therapeutic agents including chemotherapeutics, multidrug resistance modulators, antiangiogenic drugs, and cytotoxic drugs [30, 42].

Normally, if antiangiogenic drugs and chemotherapy medications are administered simultaneously, resistance may occur due to the antiangiogenic medication causing poor transport of the chemotherapeutics. This can be overcome by using a polymeric nanoparticle construct. An example is the combined loading of a single polymeric nanoparticle with DOX, a pro-apoptotic agent, and combretastatin A4, an antiangiogenic therapy. This combination treatment would cause the tumor vasculature to be diminished, inhibiting the cancerous cells from actively proliferating through the use combretastatin A4 while DOX would induce apoptosis because of its ability to effectively interact with the DNA of the tumor cells. Because of the simultaneous delivery of the two drugs, DOX was delivered to the tumor effectively without dilution in the blood stream, while combretastatin A4 was effectively delivered to the tumor vasculature. These polymeric nanostructures were still present in the tumor vasculature after 5 hours and in the tumor core after 24 hours. Finally, when compared to the loading of the same molecules in a liposomal construct, polymeric nanostructures were more effective and had better drug release kinetics showing some advantageous properties of polymeric nanoparticles over liposomes [40, 44].

A common method of drug delivery is through the use of single chain polymer linking. It has been found that by conjugating DOX and wortmannin (a kinase inhibitor) to PEG-poly(asparate hydrazide), accurate and effective loading can be achieved. This study illustrated that the molar concentrations are easily controlled when conjugating them to a polymer construct and by controlling the molar ratio of the medications, a safer, more effective form of treatment can be achieved. Data in this experiment was collected at times of 30 hours and 72 hours after the administration of the drugs. It was observed that when administered simultaneously, after 72 hours, cell viability was suppressed. Additionally, this study illustrated that this form of treatment was only effective if DOX and wortmannin were administered together. Wortmannin had very little effect alone, and the effect of DOX was significantly enhanced in the presence of wortmannin $[12,45]$.

In a 2011 study, the ratiometric control of two hydrophobic chemotherapy drugs was analyzed. To accomplish this, poly-l-lactide (PLA) was conjugated with DOX and camptothecin (CPT) - a DNA topoisomerase targeting agent, which can help to reduce cell proliferation. It was found that the ratio of DOX to CPT loaded to the polymer structure could be adjusted by simply adjusting the DOX-PLA:CPT-PLA ratio. Once loaded, an in vitro study was performed with varying concentration ratios. This study determined that this multi-drug loading method was more effective than a single-drug loaded method and that the drug payload can be more easily regulated by using the correct drug ratio. This mainly occurs due to the endocytic administering of the drugs into cancerous cells. Finally, this study illustrated that polymers have the ability to be conjugated with three or more types of drugs, allowing for the simultaneous targeting of multiple hallmarks of cancer [46].

A recent study focused on a targeted polymeric nanostructure that could be used to administer multiple therapeutic agents for colon cancer treatment. This study focused on the encapsulation of CPT and curcumin in varying ratios in a HA functionalized polymeric nanoparticle. In addition to the inhibition of cell proliferation by CPT and curcumin, curcumin is also used to block cell signaling pathways and suppress chemically induced carcinogenesis and tumor growth. Because the polymeric nanoparticles were HA functionalized, the nanoparticles also had additional biocompatibility, and this study confirmed that there was significantly increased cellular uptake efficiency in colon cancer cells with HA functionalized nanoparticles. The synthesized polymeric nanoparticles exhibited a sustained release profile and exhibited the synergistic effects of using nanoparticle combination treatments [47].

Additionally, polymeric nanoparticles have been used for combination treatments involving chemotherapeutic agents and the use of photosensitizing agents. One study focused on the dual loading of DOX and indocyanine green (ICG), an agent used for fluorescent imaging and treatment by photothermal therapy. The therapeutic agents were loaded in a PLGA-lecithin-PEG polymeric nanostructure. These polymeric nanoparticles exhibited higher temperature response, faster release of DOX upon irradiation, longer retention in tumors, and optimal imaging properties. Using laser irradiation, cancerous cells underwent apoptosis and this suppressed tumor growth which was seen in vivo, therefore exhibiting a polymeric nanostructure that can be used for both imaging and treatment purposes [48].

Polymeric micelles are a subset of polymeric nanoparticles that also offer an effective simultaneous delivery method of multiple drugs. Polymeric micelles are polymeric core-shell nanostructures that are synthesized from amphiphilic block copolymers. Since micelles are constructed like liposomes, with a hydrophilic shell 
and hydrophobic core, hydrophobic drugs may be transported in a hydrophilic manner. Also, their small size offers more efficient transport. In a 2011 study, Wang, et al., attempted to overcome multidrug resistance (MDR) with a polymeric micelle construct loaded with paclitaxel and verapamil. Paclitaxel is a mitotic inhibitor, while verapamil is used to stop p-glycoprotein, which is a key protein in drug resistance. Additionally, verapamil has shown activity against tumor promoting cells. Thus, by inhibiting drug resistance, the anti-cancer drug (paclitaxel), can be effectively administered to the tumor core. This study determined not only that drug solubility increased, but also the rate of apoptosis also increased with this new drug formulation. The apoptosis rate for the control was $4.68 \%$ while combination therapy yielded an apoptotic rate of $71.21 \%$ [49].

An additional study conducted by Shin, et al., illustrated a polymeric micelle delivery method that employed the use of three medications. Paclitaxel, 17-AAG, and rapamycin were conjugated to a PEG-b-PLA copolymer. 17-AAG works to inhibit Hsp90, a chaperone protein seen to stabilize a number of proteins required for tumor growth. Rapamycin is an immunosuppressant that can enhance immune response to tumor targeting and in combination treatments has been seen to reduce cytotoxcicity of chemotherapeutics and reduce cell resistance to treatment. Because of the hydrophobicity of these three drugs, polymeric micelle conjugation decreases the hydrophobicity of the treatment. It was determined in this study that the three-in-one loading method could deliver these cytotoxic agents safely and effectively to the tumor site. The evidence supporting this included a high tolerance of the drugs in FVB albino mice. This study also determined that the half-life of the drugs was between 1-15 hours, illustrating safe decomposition of the drug in vivo [50].

Polymeric nanoparticles and polymeric micelles have created alternate methods of drug loading, as seen in the section above. Due to the availability of many different types of polymers, various medications can be loaded into nanoparticles and then effectively released at the desired target site. Finally, by loading polymeric compounds with multiple drugs to target multiple hallmarks of cancer, more effective treatment methods can be discovered.

\section{Dendrimers}

Much like the previously described nanoparticles, dendrimers are polymeric structures that can be used to house a variety of markers and medications. As described by various reviews, dendrimers are globular, branch-like structures that are composed of synthetically constructed polymers. Dendrimers are composed of repeating units that make up an initiator core, along with multiple layers that are known as generations that can contain terminal groups [51, 52].

Because of their branched structure, dendrimers are an effective method for drug delivery [52]. This branching structure allows for the transport of both hydrophilic and hydrophobic drug molecules through the use of dendrimers. These molecules, including cytotoxic therapies, siRNA, or targeting moieties are covalently bonded to the polymeric structure of dendrimers allowing for controlled drug release $[53,54]$. These concepts will be explained further in upcoming sections.
Dendrimer Production and Loading: To achieve dendrimers' branched polymeric structures, the synthesis of monomers must occur. Various methods of production have been explored, such as click chemistry. Click chemistry refers to biocompatible reactions that often follow examples in nature in order to join substrates with biomolecules. One common example of click chemistry is copper-catalyzed Huisgen[2+3] dipolar cycloaddition. Another example of click chemistry is copper-catalyzed $[3+2]$ cycloaddition, which involves an alkyne and azide. This type of cycloaddition relies on aza-michael addition and is favorable because of the retention of hydroxyl and carboxyl groups in the dendrimer constructs [55].

One common use of dendrimers is their ability to deliver genes to tumors. As described by Dufes, et al, dendrimers are effective nanocarriers due to their high surface area to volume ratio and unique molecular architecture. In the same review, it was exhibited that when $100 \mathrm{Ag}$ HSV-tk suicide vectors are coupled with polyamidoadmine (PAMAM) dendrimers they can be used in antitumor therapy. This treatment efficiently halted the active proliferation of the tumorigenic cells [56].

Another dendrimer-based anticancer therapy involved the transport of siRNA to alter the tumor's ability to replicate. As described by Biswas, et al, siRNA can be conjugated to dendrimeric structures, due to the terminal groups present on dendrimers. These siRNA loaded dendrimers can then be used to target the complimentary mRNA present during translation. This affects the translation process in cancerous cells inhibiting their unlimited replicative potential [57].

Dendrimers can also be loaded with DOX, as described by Rouhollah, et al. PAMAM dendrimers were loaded with the cytotoxic chemotherapy medication, DOX, through the use of rotation and vibration intervals. Once loaded into the branched dendrimeric structure, stability studies were tested in PBS buffer for eight weeks and in human serum for four days. It was determined that with dendrimers, DOX could be delivered near the nucleus of a tumor cell, thus effectively treating this cancerous tumor. Dendrimers can effectively be used to load drugs and other therapeutic agents, however by using dendrimers as carriers of multiple cancer therapies, more effective treatment methods can be discovered [58].

\section{Multiple Drug Loading of Dendrimers for Treating Cancer:} The branching, globular structure of dendrimers allows for the loading of multiple drugs or targeting molecules. Hu, et al illustrated that DOX and siRNA can be dual loaded into a single dendrimer complex. DOX is used a cytotoxic agent while siRNAactively inhibits cell replication. Other combination therapies using a dendrimer complex were also briefly mentioned in this review. Many of these combination therapies involved a chemotherapeutic drug, such as DOX. This loading method further illustrates the ability of dendrimers to safely and simultaneously transport multiple cytotoxic molecules to specific tumors [12].

Dendrimers can also be loaded with targeting molecules. For example, in a study performed by Chen, et al, dendrimers were loaded with a prostate specific membrane antigen promoter, or PSMA, which is used to inhibit the expression of prostate tumor cells. These targeting dendrimers were loaded with the vi- 
ral gene vector 65-PAMAM-D, which is used to perform suicide gene therapy, leading to cancerous cells initiating cell death. This targeted combination treatment allows for the viral vector to effectively reach the tumor core in a safe manner. This form of tumor treatment was referred to as the "Bystander Effect," because no negative effects on the surrounding cells were observed. This study observed a $75 \%$ volume reduction of tumor tissue in nude mice exhibiting a targeted dendrimeric nanostructure used for inducing apoptosis [59].

Another study that incorporated multidrug delivery by use of a dendrimer complex was the delivery of DOX and pORF-htrail used to target cancer cells expressing transferrin receptors. DOX was used to target actively proliferating and dividing cells while pORF-hTRAIL was used to induce apoptosis. Transferrin receptors were targeted in this study because it was observed that transferrin receptors are 10-100 times more abundant on tumor cells. It was found by this study that less than $20 \%$ of DOX was released by diffusion, thus illustrating controlled drug release with very little premature drug leakage. Additionally, this study found that this combination method induced apoptosis in vitro and efficiently inhibited tumor growth in vivo, illustrating an effective combination treatment method that allows for sustained drug release [60].

In a 2013 study written by Acton et al, Janus dendrimers were used as carrying compounds for combination drug delivery because of their favorable bowtie configuration. In addition, this study altered the formation of generations, allowing for multiple drug loading sites on the carrier molecule. The specific drugs used in this study were benzyl alcohol, a cytotoxic agent, and 3-phenylpropionic acid, which causes tumor necrosis. To conjugate these drugs to the dendrimer, [3+2] cycloaddition of PEG-based dendrimers was performed. It was seen that after 72 hours, $85 \%$ of both drugs was released from the dendrimer in plasma exhibiting an effective drug release method that can be used for combination treatment [61].

One study conducted by Tekade, et al. illustrated the effects of $\mathrm{pH}$ on the release of drugs from a dendrimer-based nanostructure. This study specifically used all-trans retinoic acid (ATRA) and methotrexate (MTX), which can be used in combination to treat leukemia. ATRA works by binding to different receptors in order to inhibit cell growth and differentiation while MTX is used to cause folic acid deficiency in cancerous cells leading to cell death. Additionally, ATRA increases the efficacy of chemotherapeutics. It was determined that after 8 hours of incubation at physiological pH, $34 \%$ of MTX and $50 \%$ of ATRA were released, however, when incubated at $\mathrm{pH} 4,92 \%$ of MTX and $95 \%$ of ATRA were released after 8 hours of incubation. In addition, this study illustrated that the this dual-drug delivery method offered not only safe delivery of cytotoxic agents, but it offered controlled and sustained release of highly concentrated drug-loaded dendrimers. $[31,62]$.

It is clear that effective dual-loading techniques can be achieved using dendrimers, which can offer more effective targeted cancer therapies. While many of the studies described only focused on the loading of two drugs, loading dendrimers with three or more anti-cancer drugs could possibly increase the efficacy of treatments using dendrimers.

\section{Mesoporous Silica Nanoparticles}

Silicon dioxide, silica, is an oxide of silica that is found in abundance in nature as quartz, in living organisms, and is a major constituent of sand. Additionally, silica can be artificially synthesized for a variety of purposes including aerogels, artificial implants, and nanomedicine. Silica is known to be biocompatible, and allows for the encapsulation and gradual release of therapeutic agents. Silica is also used in many nanoshell structures and is used to enhance the biocompatibility of other nanostructures. Additionally, silica has the ability to have a variety of functionalizing agents conjugated to its surface making silica an optimal material for nanoparticle drug delivery.

The most common type of silica nanoparticles used for drug delivery applications are mesoporous silica nanoparticles which are porous structures composed of empty channels called mesopores. Mesoporous silica nanoparticles have high surface area, large pore volume, tunable pore size, good chemical and thermal stability due to their rigid framework, and have the ability to encapsulate large amounts of therapeutic agents due to their porous structure. Additionally, mesoporous silica nanoparticles have two surfaces, an internal and external surface, that can be functionalized with different targeting moieties for a targeted treatment method. Mesoporous silica nanoparticles can also be conjugated with "gatekeeper" molecules or nanoparticles that can regulate the premature release of drugs from the mesopores, making mesoporous silica nanoparticles have very little premature release of cytotoxic therapeutic agents. These characteristics of mesoporous silica nanoparticles make them an ideal option for nanoparticle drug delivery carriers [20].

The two most common types of mesoporous silica nanoparticle structures are MCM-41 and SBA-15. MCM-41 type nanoparticles have hexagonal mesopores with a pore size of between 1.6 to 10 nanometers and can be synthesized with sizesless than 100 nanometers. SBA-15 type nanoparticles have larger hexagonal pore sizes, and thicker pore walls, but are difficult to synthesize with sizes less than $200 \mathrm{~nm}$ making their in vivo applications limited [63]. The synthesis of the variety of mesoporous silica nanoparticles will be discussed further in the next section.

Because of the unique porous structure of mesoporous silica nanoparticles they serve as an efficient drug delivery carrier that can be mono-loaded, or multi-loaded with a variety of therapeutics or other nanostructures, and then functionalized with a targeting moiety for a targeted combination treatment that could be used to improve the efficacy of treatment methods.

\section{Mesoporous Silica Nanoparticle Production and Loading:} Mesoporous silica nanoparticles can be synthesized by a variety of methods depending on the desired morphology, pore size, and overall size of the nanostructure desired. The most common synthesis method involves the reaction of a silica precursor in the presence of an alkali catalyst and a surfactant that serves as the template for the synthesis of the mesoporous structure. Depending on the desired structure, tetraethyl orthosilicate (TEOS), sodium metasilicate, or (3-Mercaptopropyl) trimethoxysilanecan be used asthe silica precursor, and most commonly cetyltrimethylammonium bromide (CTAB) or polyoxyethylenetert-octylphenylether are used as the surfactants. By varying the silica precursor, 
surfactant, and ratios of reactants, the size of the nanoparticles, pore sizes, and morphology of resulting mesoporous silica nanoparticle can be varied. Silica nanoparticles can then be functionalized with a variety of functionalization agents, most commonly through the methods of co-condensation or grafting of the functional group on the surface of the silica nanoconstruct $[20,63$, 64].

One of the key characteristics of mesoporous silica in drug delivery is the unique porous structure that allows for a controlled drug release and a large uptake of chemotherapeutics. One study focused on the effect of pore size on the release of the chemotherapeutic, DOX. The study determined that in addition to the effective drug loading and controlled drug release characteristics of mesoporous silica there was also a pore-size-dependent characteristic of drug release. It was found that in MDF-7/ADR cancer cells, there was a greater cellular uptake and accumulation of DOX in vitro with larger pore sizes, and there was an increase in the speed of drug release corresponding to an increase in pore size. Due to this increased speed and cellular uptake, there was a decrease in multidrug resistance. This study effectively showed the relationship between pore size and drug release, and demonstrated the use of mesoporous silica nanoparticles as an effective drug delivery carrier [65].

By surface modification, mesoporous silica nanoparticle efficacy can drastically increase. A study conducted by Lu et al., focused on the conjugation of CPT loaded mesoporous silica nanoparticles with folic acid. This study found that in human pancreatic cancer xenografts on different mouse species, there was increased tumor suppression effects of CPT loaded mesoporous silica compared to free CPT. Additionally, it was observed that there was a drastic improvement in the tumor inhibiting effects of CPT loaded mesoporous silica when functionalized with folic acid. This confirms the increased efficacy of mesoporous silica when functionalized with surface agents and illustrates the biocompatibility of these nanostructures and possibilities of mesoporous silica nanoparticles as effective drug delivery carriers [66].

Another article focused on the size reduction of mesoporous silica nanoparticles to maximize the EPR effect in addition to surface functionalization for the use of mesoporous silica nanoparticles in passive targeting. The study confirmed that by reducing the size of DOX loaded mesoporous silica nanoparticles from 100 to 50 nanometers and functionalizing with a polyethyleneiminepolyethylene glycol copolymer, there was an increase in the passive delivery of drugs in a human squamous carcinoma xenograft in nude mice. In addition, this study determined that by encapsulating cytotoxic DOX in a mesoporous silica nanoconstruct that there was a reduction in systemic side effects compared to free DOX [67].

These are just some examples of mono-loaded mesoporous silica nanoparticles. The efficacy of treatment using mesoporous silica can increase when these nanoconstructs are loaded with multiple therapeutic agents in which multiple characteristics of cancer can be targeted simultaneously. Some multi-loaded methods are discussed in the next section.

Multiple Drug Loading of Mesoporous Silica Nanoparticles: Mesoporous silica nanoparticles do have the ability to be loaded with multiple therapeutics to simultaneously target multiple hallmarks of cancer, but recently the research of mesoporous silica nanoparticles has shifted into incorporating them into nanohybrid constructs. In a further section, these nanohybrid constructs will be described in detail, including nanohybrids that involve multi-loaded mesoporous silica nanoparticles. Therefore, this section will focus on only a couple of key articles that involve the combination of DOX with siRNA to induce apoptosis and overcome multidrug resistance by using siRNA to knock down gene expression.

One article focused on the combination of a DOX and Bcl-2 siRNA loaded mesoporous silica nanoparticle that was used to increase chemotherapy efficacy by stopping nonpump resistance and efflux pump resistance. Pump and nonpump resistance is initiated when chemotherapeutics trigger apoptosis and these resistances must be overcome in order to increase chemotherapy efficacy. This is achieved through the use of Bcl-2 siRNA which interferes with the $\mathrm{Bcl}-2$ protein which is seen to activate nonpump resistance. This study determined that siRNA and DOX loaded mesoporous silica were 132 times more efficient compared to free DOX. This study illustrates one example of mesoporous silica nanoparticles loaded with siRNA and DOX to increase the efficacy of chemotherapy [68].

Two other articles focused on the combination of DOX and PGlycoprotein siRNA inside a mesoporous silica nanoconstruct. It has been seen that P-glycoprotein is one of the main reasons for multidrug resistance. Therefore by targeting this protein the efficacy of cancer treatments can increase. One study used DOX/ siRNA loaded mesoporous silica functionalized with a polyethylenimine-PEG copolymer in a MCF-7/MDR xenograft model in nude mice and found that inhibition of tumor growth was increased compared to the nanostructure loaded with each molecule by itself. Using biopsies it was also determined that there was a significant knockdown of P-glycoprotein and increased efficacy of treatment [69].

The other study used a similar dual loaded mesoporous silica structure only functionalized the surface of the mesoporous silica with a phosphonate group which allows for electrostatic binding of DOX to the porous interior and polyethylenimine to the exterior surface. In a KB-V1 cancer cell line it was seen that that the intracellular and intranuclear drug concentration levels increased compared to free DOX by the effective knockdown of P-glycoprotein [70].

These studies illustrate the dual loading capability of mesoporous silica nanoparticles, and although only a few examples are demonstrated, in the next section more dual loaded mesoporous silica nanoparticles will be discussed in a nanohybrid construct.

\section{Other Potential Nanostructures and Nanohybrids}

In addition to liposomes, polymers, dendrimers, and silica nanoparticles there a variety of other nanostructures can be used in the treatment of cancer. Some of these additional nanostructures include niosomes, quantum dots, single-wall carbon nanotubes, metallic nanoparticles, and nanohybrids which combine the use of multiple nanoconstructs into one structure. The production and applications of these nanostructures will be briefly discussed 
in the following sections.

Niosomes: Niosomes, are similar to liposomes in that they are composed of a bilayer, but have some additional features that may increase efficacy in drug delivery compared to liposomes. Niosomes are unilamellar or multilamellar bilayer vesicles that are self-assembled from nonionic surfactants. In aqueous solution, like liposomes, hydrophobic regions of the surfactants remain on the inside of the bilayer, while the hydrophilic regions face outwards allowing for the encapsulation of both hydrophilic and hydrophobic drug molecules. Advantages of niosomes over liposomes are due to the lower cost of synthesis, lower toxicity, and increased accessibility and stability. The additional stability of niosomes over liposomes is due to the use of surfactants compared to the use of phospholipids in liposomes. Also, because less cholesterol is present in niosomes compared to liposomes, there is increased drug encapsulation in niosomes. Finally, niosomes tend to be smaller in size than liposomes allowing for increased cellular uptake. These similarities to liposomes and enhanced properties make niosome popular drug delivery carriers [71, 72].

Niosomes can be categorized into three classes: small unilamellar vesicles, large unilamellar vesicles, and multilamellar vesicles. All classes of niosome are synthesized by the combination of a nonionic surfactant (usually of the alkyl or dialkyl polyglycerol ether class) with cholesterol. Cholesterol allows for the rigid and spherical structure of the noisome vesicles. Common synthesis methods include the hand shaking method, reverse phase evaporation, ether injection, membrane extrusion, bubble method, or sonication. Factors that affect the resulting niosome include the type of surfactant used, the hydration temperature, and the nature of the drug being used. These various synthesis methods and variety of materials need to be considered when synthesizing a niosome for drug delivery purposes $[71,72]$.

One study focused on the drug loading of niosomes with hydroxycamptothecin (HCPT), a CPT derivative that has been shown to reduce cell proliferation. In addition to being loaded with HCPT the surface of the synthesized niosomes were also PEGylated and modified with transferrin, which is used to target the overexpression of transferrin receptors seen in cancerous cells. These synthesized niosomes proved more effective compared to free HCPT, HCPT loaded niosomes, and PEGylated HCPT loaded niosome, with an inhibition rate of $71 \%$ against $\mathrm{S} 180$ cancerous cells in mice. This study shows the applications of niosomes as well as the surface modifications that can be implemented in order to improve efficacy [73].

An additional study showed the ability for niosomes to be loaded with more than one drug for simultaneous targeting of multiple hallmarks of cancer. The study used a combination of the surfactant span 60 and cholesterol to synthesize niosomes encapsulated with the chemotherapeutic cisplatin and theophylline, a member of the xanthine family. The chemotherapeutic cisplatin works to damage cancerous DNA in order to stop cell replication while it has been seen that drugs from the xanthine family have anti-metastatic effects. This study showed that cisplatin loaded alone in a niosomal construct had increased effects compared to free cisplatin and that the combination of cisplatin and theophylline had increased reduction in number of lung nodules and secondary tumor growth compared to free cisplatin, free theo- phylline, and the mono-loading of both cisplatin and theophylline in niosomal constructs. These results were exhibited in a murine B16F10 melanoma model. Additionally, it was observed that the cytotoxic effects of cisplatin, including weight loss and bone marrow toxicity, were drastically decreased when cisplatin was loaded in a niosomal construct. This study effectively shows that niosomal nanoparticles can be used to load multiple drugs for a combination cancer treatment method [74].

As seen in the above studies, niosomes offer the ability to be loaded with multiple drugs and functionalized with different targeting moieties for combination treatment methods that can simultaneously target multiple hallmarks of cancer.

Quantum Dots: Quantum dots are nanocrystals usually synthesized from a metal such as cadmium, indium, lead, or zinc and have been viewed as an attractive nanostructure for imaging purposes due to their ability to be tuned from the visible light to infrared light spectra. Additionally, quantum dots also have the ability to be conjugated with PEG or poly [ethylene oxide] to increase their bioavailability and solubility. Also, such a coating gives these nanoparticles the ability to be conjugated with proteins, aptamers, or ligands, which can be used for targeting or treatment of specific tumors. Some specific conjugates include heat-shock proteins, antibodies which prevent HER2, and prostate specific antigens [40]. Through hybridizing quantum dots, multiple drugs can be loaded into a quantum dot in order to treat cancer in a simultaneous fashion.

One study focused on the drug loading offolate functionalized, chitosan stabilized zinc oxide quantum dots with DOX for cancer treatment applications. This study found that the synthesized zinc oxide quantum dots exhibited strong blue emission, exhibiting optimal fluorescent imaging properties of quantum dots. It was also found that the use of chitosan during the nucleation of the quantum dots increased the stability due to its hydrophilicity and cationic charge. Finally, by functionalizing the chitosan stabilized quantum dots with folate to target overexpressed folate receptors, and loading the quantum dots with DOX, these zinc oxide quantum dots showed optimal properties for targeted cancer treatments. This study illustrated the fluorescent properties of quantum dots for imaging purposes, and the ability for quantum dots to be drug loaded and functionalized with targeting moieties allowing for a targeted drug delivery method [75].

An additional study on quantum dots showed that quantum dots can be used for cancer imaging, therapy, and sensing through bifluorescence resonance energy transfer. The synthesized fluorescent quantum dots were surface functionalized with an aptamer (A10 RNA aptamer) that is used to target the prostate specific membrane antigen. The quantum dots were also intercalated with DOX creating a targeted treatment method that could also be used for fluorescent imaging of prostate cancer cells. It was observed that these multifunctional quantum dots can successfully deliver DOX to the targeted tumor and sense the delivery of DOX through the activation of the fluorescence of the quantum dots by fluorescence resonance energy transfer. This study shows the multifunctional uses of quantum dots for both cancer imaging and treatment [76].

Quantum dots have recently gained popularity due to their opti- 
mal imaging and drug loading properties, above are only a few of the articles that demonstrate the uses of quantum dots for both imaging and treatment purposes.

Single-Wall Carbon Nanotubes: Single-wall carbon nanotubes (carbon allotropes) have also been gaining popularity as drug delivery carriers due to their $\mathrm{sp} 2$ hybridized carbon surfaces and large surface areas as a result their cylindrical shape. This high surface area allows for a high capacity of drug loading both inside and on the surface of carbon nanotubes. Carbon nanotubes are also easily taken up by cells and are effective in passive targeting via the EPR effect. Additionally, carbon nanotubes have the ability for a wide variety of surface molecules to be attached to them due to carbon's versatile properties. Finally, single-wall carbon nanotubes also have both fluorescent and photothermal properties making these nanostructures applicable for imaging and treatment without any modifications. The large surface area of these carbon allotropes and easy surface modification, along with imaging and treatment properties make single-wall carbon nanotubes effective drug delivery carriers [77, 78].

Numerous studies have been conducted that combine carbon nanotubes with another treatment method, such as a chemotherapeutic, for a combination treatment that allows for drug delivery and photothermal therapy. One such study was conducted by Wang, et al., and focused on the loading of PEG coated singlewall carbon nanotubes with anti-CTLA-4 therapy for the inhibition of cancer metastasis. It was observed that when functionalized with PEG, carbon nanotubes exhibited greater stability in a variety of solutions including cell medium and serum. Additionally, the PEG functionalized carbon nanotubes were shown to be successful in photothermal destruction, which was seen to release tumor antigens, and promote the maturation of dendritic cells and production of anti-tumor cytokines. In combination with the anti-CTLA-4 therapy the PEG functionalized carbon nanotubes were seen to successfully inhibit tumor growth in both a subcutaneous tumor and metastasis model. This study exemplifies both the drug loading and photothermal effects of a PEG functionalized single-wall carbon nanotube [79].

An additional study focused on the loading of polyethylenimine functionalized single-wall carbon nanotubes with siRNA for a treatment method that delivers siRNA to cancerous cells and has the ability to destroy cancerous cells through the use of photothermal therapy. The polyethylenimine functionalized carbon nanotubes effectively inhibited cell proliferation, and induced apoptosis while being able to effectively cross the cell membrane in vitro. In vivo there was a significant amount of accumulation at the tumor site and low cytotoxicity was observed when carbon nanotubes were used for the delivery of siRNA. When combined with photothermal therapy, the single-wall carbon nanotubes had increased therapeutic efficacy. The above studies illustrate the natural photothermal effects of carbon nanotubes and their ability to be drug loaded and surface functionalized for increased efficacy [80].

Another study exemplified the biocompatibility and self imaging properties of single-wall carbon nanotubes while drug loading them with DOX. The study used DOX loaded single-wall carbon nanotubes in vivo on SMMC-7721 tumor-bearing mice and demonstrated that there is increased efficacy of DOX in carbon nanotubes compared to free DOX. The study found that some explanations for this increased efficacy are due to the ability of carbon nanotubes to accumulate at the tumor site due to the EPR effect (there was even greater accumulation when folic acid was used as a targeting agent), as well as the ability of carbon nanotubes to easily penetrate the cell membrane, and it was determined that the release of DOX was $\mathrm{pH}$ triggered reducing any premature drug release. This study illustrated not only the applications of single-wall carbon nanotubes but also delved into the reasons as to why some of the treatment results were occurring with the use of carbon nanotubes [78].

The above studies only illustrate a few of the applications and optimal properties of single-wall carbon nanotubes for the simultaneous targeting of multiple hallmarks of cancer.

Metallic Nanoparticles: Metallic nanoparticles have also become increasingly popular in cancer treatment methods due to their optimal properties for photothermal therapy or magnetic hyperthermia. Specifically, gold nanoparticles have become of particular interest in cancer treatment due to their robustness, biocompatibility, biodegradability, and their lack of thermal denaturing [40]. Additionally, since gold nanoparticles are metallic in nature they can be used to deliver energy in the form of heat to unwanted tumor cells through the use of photothermal therapy and surface plasmon resonance [81]. Gold nanoparticles also have the ability to have targeting agents or drugs attached to the surface, such as PEG-thiols or chemotherapeutic agents, that can be used to synthesize a targeted treatment method.

Gold nanoparticles can be synthesized in a variety of sizes from 2 $\mathrm{nm}$ to $100 \mathrm{~nm}$, and most synthesis methods involve the reduction of a gold chloride salt $\left(\mathrm{HAuCl}_{4}\right)$ into colloidal gold by a reducing agent. The most common gold synthesis method is the Turkevich method which results in gold nanoparticles of sizes between 20 and $40 \mathrm{~nm}$. The Turkevich method involves the reduction of $\mathrm{HAuCl}_{4}$ by a sodium citrate resulting in the formation of 20-40 nm gold colloids [82]. Another common method for producing sub-5nm gold colloids (useful for gold nanoshell synthesis) is the Duff method which involves the reduction of $\mathrm{HAuCl}_{4}$ by tetrakis (hydroxymethyl) phosphonium chloride. There are a variety of other gold nanoparticle synthesis methods that can be used depending on the desired size and surface stabilizer of the colloidal gold nanoparticles [83].

Although metallic nanoparticles have been successful for photothermal therapy and magnetic hyperthermia, it is difficult for drug molecules to be conjugated to metallic nanoparticles. Therefore, in order to synthesize a combination treatment method, metallic nanoparticles can be incorporated with another nanoparticle delivery system, such as a polymer, liposome, or silica construct. This is often referred to as a nanohybrid construct. Nanohybrid constructs, discussed below, have become increasingly popular because of their ability to combine a variety of treatment methods to target multiple hallmarks simultaneously.

Nanohybrids: Nanohybrids involve combining multiple nanoconstructs into one cohesive structure. One such example was described in a study that focused on a nanohybrid treatment in which liposomes loaded with a photosensitizing agent were combined with metallic or magnetic nanoparticles. This allowed for 
combined photodynamic therapy and photothermal therapy or magnetic hyperthermia treatment. These liposomes were synthesized with either magnetic or metallic nanoparticles encapsulated in the liposome, and with a photosensitizer agent in the lipid bilayer. The purpose of using magnetic nanoparticles would be to destroy cancerous cells through the use of heat produced by magnetic hyperthermia, whereas if metallic nanoparticles were used, photothermal therapy could be used to produce heat and kill cancerous cells. The purpose of the photosensitizer agent would be for the use of PDT. Depictions of PDT, photothermal therapy, and magnetic hyperthermia can be seen in Figure 4. This method shows a combination treatment involving multiple nanostructures used for either magnetic hyperthermia or photothermal therapy, in combination with PDT. This method achieved complete cancer cell death in vitro and solid-tumor ablation in an in vivo rodent model through the triggering of apoptotic signaling pathways. This study shows the viability of combination treatments without the use of any cytotoxic drug loading [84].

Another nanohybrid involving metallic nanoparticles are metal nanoshells. One of the most common metallic nanoshells is a nanoshell that involves a dielectric silica core and a thin gold shell. Because of the dielectric silica core, these nanoshells have shown a red-shifted absorption compared to free gold nanoparticles. This red-shift of the absorption into the near infrared region between 600 and 800 nanometers allows for maximum tissue penetration when performing photothermal therapy on the nanoshells. This red-shift is one of the reasons a metal nanoshell hybrid is more effective than free metallic nanoparticles [85]. Another recent article focused on the synthesis of gold nano matryoshkas, which involve a gold nanoparticle core, with a silica shell, followed by the formation of another gold shell on the surface. Compared to normal silica-gold core-shells these nano matryoshkas absorbed more light from photothermal therapy and more efficiently reduced tumor size and reoccurrence in triple negative breast cancer bearing mice [86]. These are just a few examples of how metallic nanohybrids can be used to combine multiple treatments and increase the efficacy of treatment methods.

In addition to the combination of metal nanoparticles with other nanoparticles, there have been studies that have used combinations of lipids, polymers, dendrimers, and silica nanoparticles to create efficient nanohybrid treatment methods. A 2016 study conducted by Liu, et al. focused on a lipid coated mesoporous silica nanoparticle for the treatment of pancreatic cancer. The chemotherapeutic, irinotecan, which is commonly used in treatment of pancreatic cancer, was loaded inside a lipid coated mesoporous silica nanoparticle. It was found that by having a lipid coated silica nanoparticle there was less drug leakage, increased drug concentrations at tumor sites, more efficiency in reducing tumor metastasis, and reduced gastrointestinal, bone marrow, and liver cytotoxicity [87].

A similar study used a lipid coated mesoporous silica nanoparticle for treatment of pancreatic cancer. The mesoporous silica nanoparticle was dual loaded with gemcitabine and paclitaxel. It was seen that in this dual loaded structure that the effects of gemcitabine were more prominent due to the suppression of tumor stroma and inhibition of the gemcitabine inactivating enzyme due to the effects of paclitaxel. It was also found that a higher drug loading could be achieved by using a lipid bilayer on the surface. In mice bearing pancreatic cancer tumors (PANC-1 xenografts) it was found that dual loaded mesoporous silica was more effective in reducing tumor size compared to the combination of free drugs, and mono-loaded mesoporous silica. Additionally, it was found that there was no local or systemic cytotoxicity indicating a lack of premature drug release. This study exemplifies a dual loaded nanohybrid that can be used as a safe and efficient drug delivery carrier [88].

An additional study used a mesoporous silica nanoparticle conjugated with polymer based molecules on the surface that served

Figure 4. Remotely triggered cytotoxic treatments. (A) Photodynamic therapy, which uses light radiation and a photosensitizer to convert reactive oxygen into a radical oxygen species (ROS) that is used to kill cancerous cells. (B) Photothermal therapy, which uses light radiation and surface plasmon resonance to produce heat from a metallic nanoparticle that is used to kill cancerous cells. (C) Magnetic hyperthermia, which uses magnetic radiation to produce heat from a magnetic nanoparticle that is used to kill cancerous cells.

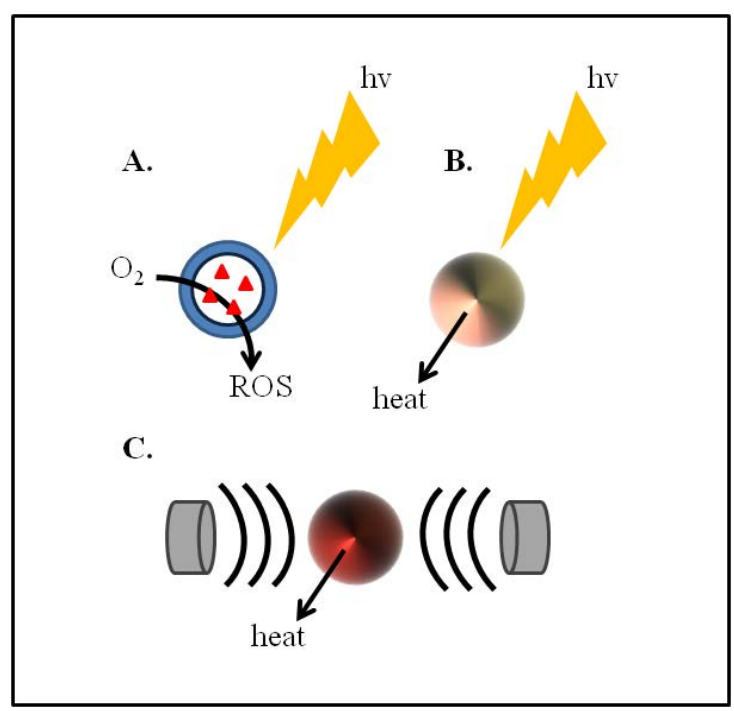


as "gatekeepers" for drug release from mesoporous silica. The study illustrated that at a low temperature (4 degrees Celsius) the nanohybrids could be loaded with a single molecule, or multiple molecules due to the open conformation of the polymers at that temperature. When the nanohybrids are placed at physiological temperature (37 degrees Celsius) the polymer collapses, blocking any premature cytotoxic drug release. When the nanohybrids accumulated in the tumor site, ultrasound irradiation was used to change the hydrophobicity of the polymers changing the polymer structure to a coil-like structure, allowing for drug release from the nanohybrid. This study illustrated a nanohybrid that allowed for remote triggering of drug release through the use ultrasound irradiation using a mesoporous silica core conjugated with polymeric structures on the surface [89].

There continue to be developments in the variety and uses of nanoparticles and nanohybrids for combination treatment methods and this article serves to introduce some of the common types of nanoparticle structures and the endless applications they can be used for, specifically combination treatments for simultaneous targeting of multiple hallmarks of cancer.

\section{Conclusions and Future Considerations}

Traditional cancer therapies are not only harmful to the patient, but they also yield poor survival rates because of the cytotoxic nature of many normal treatments. To improve the efficacy of cancer treatment methods, nanoparticles have been developed to safely and efficiently deliver drugs to the tumor site. Nanoparticles allow for the transport of both hydrophobic and hydrophilic molecules and because of their size, they can take advantage of the tumor vasculature to administer drug molecules to the tumor core. By using dendrimers, liposomes, polymers, silica nanoparticles and a variety of other nanoparticles and nanohybrids, cytotoxic medications can be safely and efficiently delivered to the tumor core. Additionally, nanoparticles can be used in combination with other therapies, such as a VEGF inhibitor, or a photosensitized treatment method, to improve the efficacy of treatments.

By combining multiple therapies into one nanostructure a more effective treatment can be developed that can target multiple characteristics of cancer simultaneously. For example, a liposomal construct could be loaded with a VEGF inhibitor to reduce angiogenesis, DOX to induce apoptosis, and siRNA to limit the replicative potential of cancer cells. This liposomal nanoconstruct could also be loaded with a photosensitizer or metallic nanoparticle for use in PDT, photothermal therapy, or magnetic hyperthermia. This hypothetical nanoparticle could then be coated with PEG giving it the biocompatibility to travel safely through the body. Finally, this nanoparticle could be conjugated with targeting molecules on the surface such as ligands or aptamers in order to increase drug delivery efficacy to the tumor site. This is just one example of the multitude of possibilities that can be achieved through the use of nanoparticles to create an effective targeted treatment method.

There are numerous other nanomedicine treatment methods being developed, and many organizations have shown interest in nanoparticles as drug delivery carriers, such as Abraxis, BIND Therapeutics, and Pharmaco-Kinesis Corporation. Additionally, there have been significant clinical advancements in nanomedi- cine including the approval of nanomedicine drugs such as Abraxane, DaunoXome, and Doxil. This interest in nanomedicine is continuing to push the envelope of new cancer treatments, leading to more efficient and safer treatment methods.

As seen, nanoparticle combination treatment therapies have shown promise in the targeting of multiple hallmarks of cancer, but in order to improve efficacy even more these nanostructure must be examined in greater detail in vivo. By focusing on in vivo studies it can be determined what hallmarks of cancer must be targeted in what order for the highest efficiency in reducing tumor size, growth, and reoccurrence. By formulating the correct combination of hallmark-targeting therapeutics within a hybrid nanoparticle construct, combination nanoparticle treatment methods could prove to be an effective means of targeting multiple hallmarks of cancer and could help increase the current cancer survival rate.

An ideal nanoparticle-based combination regimen will accomplish synergistically enhanced treatment outcome without exhibiting any additive toxicities due to the two or more drugs used in the combination. However, such nanoparticle-based combinations should be based on simple, easy to reproduce, scalable manufacturing processes with negligible batch-to-batch variations. Next-generation combination nanomedicines will reduce toxicity, enhance efficacy, and overcome drug resistance with improved therapeutic indices to elicit sustained treatment responses towards improving survival and quality of life in cancer patients.

\section{Acknowledgments}

The authors acknowledge funding from the National Cancer Institute, a part of the National Institutes of Health (Award \# R00CA153948).

\section{References}

[1]. National Cancer Institute (2016) Cancer Statistics.

[2]. American Cancer Society, Cancer Facts \& Figures 2016.

[3]. Hanahan D, Weinberg RA (2000) The hallmarks of cancer. Cell 100(1): 57-70.

[4]. Hanahan, Douglas, and Robert A. Weinberg (2011) Hallmarks of cancer: the next generation.Cell 144(5): 646-674.

[5]. UdagawaT, Wood M (2010) Tumor-stromal cell interactions and opportunities for therapeutic intervention.Current Opinion in Pharmacology 10(4): 369-374.

[6]. Trevor AJ, Katzung BG, Master SB (2010) Katzung and Trevor's Pharmacology \& Board Review; $9^{\text {th }}$ Ed. McGraw Hill.

[7]. Prabhakar U, Maeda H, Jain RK, Sevick-Muraca EM, Zamboni W, et al. (2013) Challenges and key considerations of the enhanced permeability and retention effect (EPR) for nanomedicine drug delivery in oncology.Cancer Research 73(8): 2412-2417.

[8]. Floor SL, Dumont JE, Maenhaut C, Raspe E. et al., (2012) Hallmarks of cancer: of all cancer cells, all the time? Trends in Molecular Medicine 18(9): 509-515.

[9]. Folkman J, Kalluri R (2004) Cancer without disease. Nature427 (6977): 787-787.

[10]. Sengupta S, Eavarone D, Capila I, Zhao G, Watson N, et al. (2005) Temporal targeting of tumour cells and neovasculature with a nanoscaledelivery system. Nature 436(7050): 568-572.

[11]. Green MR1, Manikhas GM, Orlov S, Afanasyev B, Makhson AM, et al. (2006) Abraxane ${ }^{\oplus}$, a novel Cremophor ${ }^{\oplus}$-free, albumin-bound particle form of paclitaxel for the treatment of advanced non-small-cell lung cancer.Annals of Oncology 17(8): 1263-1268.

[12]. Hu CM, Aryal S, Zhang L (2010) Nanoparticle-assisted combination therapies for effective cancer treatment. Therapeutic Delivery 1(2): 323-334.

[13]. V. Roy1, B. R. LaPlant, G. Gross, C. L. Bane, F. M. Palmier et al. (2009) 
Phase II trial of weekly nab (nanoparticle albumin-bound)-paclitaxel (nabpaclitaxel)(Abraxane $e^{\circ}$ in combination with gemcitabine in patients with metastatic breast cancer (N0531)..Annals of Oncology 20(3): 449-453.

[14]. National Cancer Institute, Photodynamic Therapy for Cancer, 2016.

[15]. Sano K, Takahito Nakajima, Peter L. Choyke, and Hisataka Kobayashi (2012) Markedly Enhanced Permeability and Retention Effects Induced by Photo-immunotherapy of Tumors.ACS Nano 7(1): 717-724.

[16]. Bertrand N, Wu J, Xu X, Kamaly N, Farokhzad OC (2014) Cancer nanotechnology: The impact of passive and active targeting in the era of modern cancer biology. Advanced Drug delivery Reviews 66: 2-25.

[17]. Shukla S, DiFranco NA, Wen AM, Commandeur U, Steinmetz NF (2015) To Target or Not to Target: Active vs. Passive Tumor Homing of Filamentous Nanoparticles Based on Potato virus X. Cellular and Molecular Bioengineering 8(3): 433-444.

[18]. Goldberg MS, et al., (2013) Biotargeted nanomedicines for cancer: six tenets before you begin. Nanomedicine 8(2): 299-308.

[19]. Joshi M, Gaurav T, Ruchi T, Birendra S. (2012) Nanomedicine to improve drug delivery outcomes. Chronicles of Young Scientists 3(4): 258.

[20]. Slowing II, Vivero-Escoto JL, Wu C, Lin VSY (2008) Mesoporous silica nanoparticles as controlled release drug delivery and gene transfection carriers. Advanced Drug Delivery Reviews60(11): 1278-1288.

[21]. Hall RM, Sun T, Ferrari M (2012) A Portrait of Nanomedicine and Its Bioethical Implications. The Journal of Law, Medicine \& Ethics40(4): 763779 .

[22]. Colas J, Shi W, Rao VS, Omri A, Mozafari MR, Singh H (2007) Microscopical investigations of nisin-loaded nanoliposomes prepared by Mozafari method and their bacterial targeting.Micron38(8): 841-847.

[23]. Hwang T, et al. (2011) Fabrication of nano-scale liposomes containing doxorubicin using Shirasu porous glass membrane. Colloids and Surfaces A: Physicochemical and Engineering Aspects 392(1): 250-255.

[24]. Malam Y, Loizidou M, Seifalian AM (2009) Liposomes and Nanoparticles: Nanosized Vehicles for Drug Delivery in Cancer. Trends in Pharmacological Sciences 30(11): 592-599.

[25]. Immordino ML, Dosio F, Cattel L (2006) Stealth liposomes: review of the basic science, rationale, and clinical applications, existing and potential. International Journal of Nanomedicine 1(3): 297.

[26]. Han N-K, Shin DH, Kim JS, Weon KY, Jang C-Y, et al. (2016) Hyaluronanconjugated liposomes encapsulating gemcitabine for breast cancer stem cells. International Journal of Nanomedicine. 11:1413-1425.

[27]. Daily Med, Current Medical Information, Doxil (doxorubicin hydrochloride), U.S. National Library of Medicine, 2016.

[28]. eMC, DaunoXome Injection $2 \mathrm{mg} / \mathrm{ml}$ Concentrate for Solution for Infusion, 2016.

[29]. Ichikawa K, Hikita T, Maeda N, Yonezawa S, Takeuchi Y, et al. (2005) Antiangiogenic photodynamic therapy (PDT) by using long-circulating liposomes modified with peptide specific to angiogenic vessels.BiochimicaetBiophysicaActa (BBA) - Biomembranes 1669(1): 69-74.

[30]. Hu CM, Zhang L (2012) Nanoparticle-based combination therapy toward overcoming drug resistance in cancer. Biochemical pharmacology 83(8): 1104-1111.

[31]. Parhi P, Mohanty C,Sahoo SK (2012) Nanotechnology-based combinational drug delivery: an emerging approach for cancer therapy. Drug Discovery Today 17(17): 1044-1052.

[32]. Batist G, Gelmon KA, Chi KN, Miller WH Jr, Chia SK, et al., (2009) Safety, pharmacokinetics, and efficacy of CPX-1 liposome injection in patients with advanced solid tumors. Clinical Cancer Research 15(2): 692-700.

[33]. Tangutoori S, Spring BQ, Mai Z, Palanisami A, Mensah LB, et al. (2016) Simultaneous delivery of cytotoxic and biologic therapeutics using nanophotoactivatable liposomes enhances treatment efficacy in a mouse model of pancreatic cancer. Nanomedicine: Nanotechnology, Biology and Medicine 12(1): 223-234.

[34]. Spring BQ, Bryan Sears R, Zheng LZ, Mai Z, Watanabe R, et al. (2016) A photoactivable multi-inhibitor nanoliposome for tumour control and simultaneous inhibition of treatment escape pathways. Nature Nanotechnology11(4): 378-387.

[35]. Chang R (2008) General Chemistry: The Essential Concepts. McGraw-Hill.

[36]. Iyer AK, Morrissey DV, Amiji MM, Ganesh S (2013) Hyaluronic acid based self-assembling nanosystems for CD44 target mediated siRNA delivery to solid tumors.Biomaterials 34(13): 3489-3502.

[37]. Shin-ichi Yusa, Shigeru Yamago , Makoto Sugahara, Sanae Morikawa, Tohei Yamamoto, et al., (2007) Thermo-responsive diblock copolymers of poly ( $\mathrm{N}$-isopropylacrylamide) and poly (N-vinyl-2-pyrroridone) synthesized via organotellurium-mediated controlled radical polymerization (TERP). Macromolecules40(16): 5907-5915.

[38]. Soppimath KS, Aminabhavi TM, Kulkarni AR, Rudzinski WE (2001) Biodegradable polymeric nanoparticles as drug delivery devices.Journal of Controlled Release 70(1): 1-20.
[39]. Georges V, Kazmeier H (1993) Free Radical Polymerization.Macromolecules 26: 5316

[40]. vanVlerken LE, Amiji MM (2006) Multi-functional polymeric nanoparticles for tumour-targeted drug delivery.Expert Opinion on Drug Delivery 3(2):205-216.

[41]. Huang H, Zhang X, Yu J, Zeng J, Chang PR, (2013) Fabrication and Reduction-Sensitive Behavior of Polyion Complex Nano-micelles Based on PEGConjugated Polymer Containing Disulfide Bonds as a Potential Carrier of Anti-Tumor Paclitaxel.Colloids and Surfaces B: Biointerfaces 110: 59-65.

[42]. Kukowska-Latallo JF, Candido KA, Cao Z, Nigavekar SS, Majoros IJ, et al. (2005) Nanoparticle targeting of anticancer drug improves therapeutic response in animal model of human epithelial cancer.Cancer Research 65(12): 5317-5324.

[43]. Dahlman JE, Barnes C, Khan OF, Thiriot A, Jhunjunwala Set al. (2014) In vivo endothelial siRNA delivery using polymeric nanoparticles with low molecular weight.Nature Nanotechnology9(8): 648-655.

[44]. Shiladitya Sengupta, David Eavarone,, Ishan Capila, Ganlin Zhao, Nicki Watson, et al. (2005) Temporal targeting of tumour cells and neovasculature with a nanoscale delivery system. Nature436(7050): 568-572.

[45]. Bae Y1, Diezi TA, Zhao A, Kwon GS. (2007) Mixed polymeric micelles for combination cancer chemotherapy through the concurrent delivery of multiple chemotherapeutic agents.Journal of Controlled Release 122(3): 324-330.

[46]. Aryal S, Hu C-M, Zhang L (2011) Polymeric nanoparticles with precise ratiometric control over drug loading for combination therapy. Molecular Pharmaceutics 8(4): 1401-1407.

[47]. Xiao B, et al. (2015) Hyaluronic acid-functionalized polymeric nanoparticles for colon cancer-targeted combination chemotherapy. Nanoscale 7(42): 17745-17755.

[48]. Zheng M, et al., (2013) Single-Step Assembly of DOX/ICG Loaded LipidPolymer Nanoparticles For Highly Effective Chemo-photothermal Combination Therapy. ACS Nano7(3): 2056-2067.

[49]. Wang F, et al. (2011) Synergistic effect of folate-mediated targeting and verapamil-mediated P-gp inhibition with paclitaxel-polymer micelles to overcome multi-drug resistance. Biomaterials32(35): 9444-9456.

50]. Shin H-C, et al. (2011) A 3-in-1 polymeric micelle nanocontainer for poorly water-soluble drugs. Molecular Pharmaceutics 8(4): 1257-1265.

[51]. Zhang L, Gu FX, Chan JM, Wang AZ, Langer RS, et al. (2007) Nanoparticles in medicine: therapeutic applications and developments. Clinical Pharmacology \& Therapeutics 83(5): 761-769.

[52]. Astruc D, Boisselier E, Ornelas C (2010) Dendrimers designed for functions: from physical, photophysical, and supramolecular properties to applications in sensing, catalysis, molecular electronics, photonics, and nanomedicine. Chemical Reviews 110(4): 1857-1959.

[53]. Menjoge AR, Kannan RM, Tomalia DA (2010) Dendrimer-based drug and imaging conjugates: design considerations for nanomedical applications. Drug Discovery Today 15(5): 171-185.

[54]. Leshchiner I, Boiko N, Kumar J, Richardson R M, Muzafarov A, et al. (2013) Synthesis and physical behavior of amphiphilic dendrimers with layered organization of hydrophilic and hydrophobic blocks. Colloid and Polymer Science 291(4): 927-936.

[55]. Shen Y, Ma Y, Li Z (2013) Facile synthesis of dendrimers combining azaMichael addition with Thiol-yne click chemistry.Journal of Polymer Science Part A: Polymer Chemistry 51(3): 708-715.

[56]. Dufès C, Uchegbu IF, Schätzlein AG (2005) Dendrimers in gene delivery. Advanced Drug Delivery Reviews 57(15): 2177-2202. https://www.researchgate.net/publication/7459236_Dendrimers_in_gene_delivery_Adv_ Drug_Deliv_Rev

[57]. Biswas S, Torchilin VP (2013) Dendrimers for siRNA Delivery. Pharmaceuticals 6(2): 161-183

[58]. Rouhollah K, Pelin M, Serap Y, Gozde U, Ufuk G (2013) Doxorubicin loading, release, and stability of polyamidoaminedendrimer-coated magnetic nanoparticles. Journal of Pharmaceutical Sciences 102(6): 1825-1835.

[59]. Chen Y, Wang G, Kong D, Zhang Z, Yang K, et al. (2013) Double-targeted and double-enhanced suicide gene therapy mediated by generation 5 polyamidoaminedendrimers for prostate cancer. Molecular Carcinogenesis 52(3): $237-246$

[60]. Han L, Huang R, Li J, Liu S, Huang S, et al. (2011) Plasmid pORF-hTRAIL and doxorubicin co-delivery targeting to tumor using peptide-conjugated polyamidoaminedendrimer. Biomaterials 32(4): 1242-1252.

[61]. Acton AL, Cristina Fante, Brian Flatley, Stefano Burattini, Ian W. Hamley, et al. (2013) Janus PEG-Based Dendrimers for Use in Combination Therapy: Controlled Multi-Drug Loading and Sequential Release. Biomacromolecules 14(2): 564-574.

[62]. Tekade RK, Dutta T, Gajbhiye V, Jain NK (2009) Exploring dendrimer towards dual drug delivery: $\mathrm{pH}$ responsive simultaneous drug-release kinetics. Journal of Microencapsulation 26(4): 287-296. 
[63]. Tang F, Li L, Chen D (2012) Mesoporous Silica Nanoparticles: Synthesis, Biocompatibility and Drug Delivery. Advanced Materials 24(12): 15041534 .

[64]. Trewyn B G, Slowing II, Giri S, Chen H-T, Lin VSY (2007) Synthesis and Functionalization of a Mesoporous Silica Nanoparticle Based on the Sol-Gel Process and Applications in Controlled Release. Accounts of Chemical Research 40(9): 846-853.

[65]. Gao Y, et al., (2011) Controlled Intracellular Release of Doxorubicin in Multidrug-Resistant Cancer Cells by Tuning the Shell-Pore Sizes of Mesoporous Silica Nanoparticles. ACS Nano 5(12): 9788-9798.

[66]. Lu J, Li Z, Zink JI, Tamanoi F (2012) In vivo tumor suppression efficacy of mesoporous silica nanoparticles-based drug-delivery system: enhanced efficacy by folate modification. Nanomedicine: Nanotechnology, Biology and Medicine 8(2): 212-220.

[67]. Meng H, Xue M, Xia T, Ji Z, Tarn DY, et al. (2011) Use of Size and a Copolymer Design Feature To Improve the Biodistribution and the Enhanced Permeability and Retention Effect of Doxorubicin-Loaded Mesoporous Silica Nanoparticles in a Murine Xenograft Tumor Model. ACS Nano 5(5): 4131-4144.

[68]. Chen AM, Zhang M, Wei D, Stueber D, Taratula O, et al. (2009) Co-delivery of Doxorubicin and Bcl-2 siRNA by Mesoporous Silica Nanoparticles Enhances the Efficacy of Chemotherapy in Multidrug-Resistant Cancer Cells. Small 5(23): 2673-2677.

[69]. Meng H, Mai WX, Zhang H, Xue M, Xia T, et al. (2013) Codelivery of an Optimal Drug/siRNA Combination Using Mesoporous Silica Nanoparticles To Overcome Drug Resistance in Breast Cancer in Vitro and in Vivo.ACS Nano 7(2): 994-1005.

[70]. Meng H, Liong M, Xia T, Li Z, Ji Z, et al. (2010) Engineered Design of Mesoporous Silica Nanoparticles to Deliver Doxorubicin and P-Glycoprotein siRNA to Overcome Drug Resistance in a Cancer Cell Line. ACS Nano 4(8): 4539-4550.

[71]. Kazi KM, Asim Sattwa Mandal, Nikhil Biswas, Arijit Guha, Sugata Chatterjee, et al., (2010) Niosome: A future of targeted drug delivery systems. Journal of Advanced Pharmaceutical Technology and Research 1(4): 374-380.

[72]. Uchegbu IF,Vyas SP (1998) Non-ionic surfactant based vesicles (niosomes) in drug delivery. International Journal of Pharmaceutics 172(2): 33-70.

[73]. Hong M, Zhu S, Jiang Y, Tang G, Pei Y (2009) Efficient tumor targeting of hydroxycamptothecin loaded PEGylatedniosomes modified with transferring. Journal of Controlled Release 133(2): 96-102.

[74]. Gude RP, Jadhav MG, Rao SG, Jagtap AG. et al., (2004) Effects of NiosomalCisplatin and Combination of the Same with Theophylline and with Activated Macrophages in Murine B16F10 Melanoma Model.Cancer Biotherapy and Radiopharmaceuticals 17(2): 183-192.

[75]. Yuang Q, Hein S, Misra RDK (2010) New generation of chitosan-encapsulated $\mathrm{ZnO}$ quantum dots loaded with drug: Synthesis, characterization and in vitro drug delivery response. ActaBiomaterialia 6(7): 2732-2739.

[76]. Bagalkot V, Zhang L, Levy-Nissenbaum E, Jon S, Kantoff PW, Langer R, Farokhzad OC., et al. (2007) Quantum Dot-Aptamer Conjugates for Synchronous Cancer Imaging, Therapy, and Sensing of Drug Delivery Based on Bi-Fluorescence Resonance Energy Transfer. Nano Letters 7(10): 30653070 .

[77]. Zhang W, Zhang Z, Zhang Y (2011) The application of carbon nanotubes in target drug delivery systems for cancer therapies. Nanoscale Research Letters. 6(1): 1
[78]. Meng L, Zhang X, Lu Q, Fei Z, Dyson PJ (2012) Single walled carbon nanotubes as drug delivery vehicles: Targeting doxorubicin to tumors. Biomaterials 33(6): 1689-1698.

[79]. Wang C, et al., (2014) Immunological Responses Triggered by Photothermal Therapy with Carbon Nanotubes in Combination with Anti-CTLA-4 Therapy to Inhibit Cancer Metastasis. Advanced Materials 26(48): 8154-62.

[80]. Wang L, Shi J, Zhang H, Li H, Gao Y, et al., (2013) Synergistic anticancer effect of RNAi and photothermal therapy mediated by functionalized singlewalled carbon nanotubes. Biomaterials 34(1): 262-274.

[81]. Kyeongsoon Park, Seulki Lee, Eunah Kang, Kwangmeyung Kim, Kuiwon Choi, et al. (2009) New generation of multifunctional nanoparticles for cancer imaging and therapy. Advanced Functional Materials 19(10): 1553 1566

[82]. J Kimling, M Maier, B Okenv, V Kotaidis, A Plech, et al., (2006) Turkevich Method for Gold Nanoparticle Synthesis Revisited. The Journal of Physical Chemistry B 110(32): 15700-15707.

[83]. Duff DG, Baiker A, Edwards PP (1993) A new hydrosol of gold clusters. 1. Formation and particle size variation. Langmuir 9(9): 2301-2309.

[84]. Riccardo Di Corato, Gaëlle Béalle, Jelena Kolosnjaj-Tabi, Ana Espinosa, Claire Wilhelm, et al., (2015) Combining Magnetic Hyperthermia and Photodynamic Therapy for Tumor Ablation with Photoresponsive Magnetic Liposomes. ACS Nano 9(3): 2904-2916.

[85]. Oldenburg SJ, Averitt RD, Westcott SL, Halas NJ (1998) Nanoengineering of optical resonances. Chemical Physics Letters 288(2): 243-247.

86]. Ayala-Orozco C1, Urban C, Knight MW, Urban AS, Neumann O, et al., (2014) Au Nanomatryoshkas as Efficient Near-Infrared Photothermal Transducers for Cancer Treatment: Benchmarking against Nanoshells. ACS Nano 8(6): 6372-6381.

[87]. Xiangsheng Liu, Allen Situ, Yanan Kang, Katie Rose Villabroza, Andre E. Nel, et al. (2016) Irinotecan Delivery by Lipid-Coated Mesoporous Silica Nanoparticles Shows Improved Efficacy and Safety over Liposomes for Pancreatic Cancer. ACS Nano 10(2): 2702-2715.

[88]. Meng H, Wang M, Liu H, Liu X, Situ A, et al., (2015) Use of a Lipid Coated Mesoporous Silica Nanoparticle Platform for Synergistic Gemcitabine and Paclitaxel Delivery to Human Pancreatic Cancer in Mice. ACS Nano 9(4): 3540-3557.

[89]. Paris JL, Cabañas VM, Manzano M, Vallet-Regi M (2015) Polymer-Grafted Mesoporous Silica Nanoparticles as Ultrasound-Responsive Drug Carriers. ACS Nano 9(11): 11023-11033.

[90]. (2016) National Cancer Institute, A to Z List of Cancer Drugs.

Special Issue on

"Nanomedicine and Biotherapeutics"

Edited by:

Stanley Saamoah Moffatt, Nova Southeastern

University, USA.

E-mail: stanley.moffatt@regentghana.net 